\title{
Wrapped M5-branes and complex saddle points
}

\author{
Pietro Benetti Genolini \\ Department of Applied Mathematics and Theoretical Physics, University of Cambridge, \\ Wilberforce Road, Cambridge, CB3 OWA, U.K. \\ Department of Mathematics, King's College London, \\ Strand, WC2R 2LS, U.K. \\ E-mail: Pietro.Benetti_Genolini@kcl.ac.uk
}

\begin{abstract}
We study the effects of the introduction of a $\vartheta$ term in minimal gauged supergravity in four dimensions. We show why this term is not present in supergravity duals of field theories arising on wrapped $M 2$-branes, but is there in the case of $M 5$-branes wrapping hyperbolic manifolds $\Sigma_{3}$, and compute the higher-derivative corrections. Having proved that the on-shell supergravity action of any supersymmetric solution can be expressed in terms of data from the fixed points of a Killing vector, we show that it is proportional to a complex topological invariant of $\Sigma_{3}$. This is consistent with the characteristics of the dual three-dimensional $\mathcal{N}=2 \mathrm{SCFT}$ predicted by the $3 d-3 d$ correspondence, and we match the large $N$ limit of its partition functions in the known cases.
\end{abstract}

KEYwords: AdS-CFT Correspondence, Supergravity Models, Supersymmetry and Duality

ArXiv EPrint: 2110.15955 


\section{Contents}

1 Introduction and summary 1

2 Reduction of the supergravity action $\quad 4$

2.1 Action 4

2.2 Local form of supersymmetric solutions 6

$\begin{array}{lll}2.3 & \text { On-shell action } & 7\end{array}$

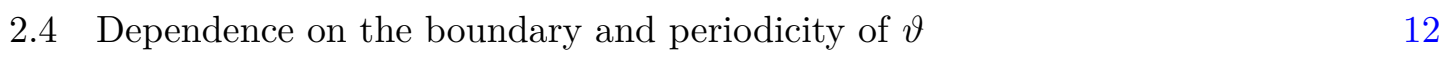

3 Examples $\quad 13$

$\begin{array}{lll}3.1 & \mathrm{AdS}_{4} & 13\end{array}$

$\begin{array}{lll}3.2 & \text { Supersymmetric black holes } & 15\end{array}$

$\begin{array}{lll}3.3 & \frac{1}{4} \text {-BPS solutions } & 15\end{array}$

$\begin{array}{lll}3.4 & \frac{1}{2} \text {-BPS solutions } & 17\end{array}$

$\begin{array}{lll}3.5 & \text { Other topologies } & 19\end{array}$

4 Origin of the $\vartheta$ term $\quad 20$

4.1 M2-branes 21

4.2 M5-branes 22

$\begin{array}{lll}4.3 & \text { Uplift: topological considerations } & 26\end{array}$

$\begin{array}{lll}4.4 & \text { Subleading corrections } & 27\end{array}$

$\begin{array}{lll}5 & \text { Field theory } & 30\end{array}$

$\begin{array}{ll}\text { A Chern-Simons conventions } & 35\end{array}$

B Bott-Cattaneo formula $\quad 38$

\section{Introduction and summary}

Four-dimensional Einstein-Maxwell theory with a negative cosmological constant describes the bosonic sector of minimal gauged supergravity [1]. Much of the recent interest in this theory is driven by the fact that its supersymmetric asymptotically locally AdS solutions $Y_{4}$ describe the gravity duals to three-dimensional $\mathcal{N}=2$ SCFTs on curved backgrounds $M_{3}$, with $\partial Y_{4} \cong M_{3}$. The holographically renormalized on-shell action of the supergravity solution $Y_{4}$ is related by the AdS/CFT correspondence to a large $N$ limit of the free energy of the dual SCFT on $M_{3}$. The converse problem is more involved: given a SCFT on $M_{3}$ with some geometric structure, there are many $Y_{4}$ filling $M_{3}$ with boundary conditions matching the geometry of $M_{3}$. It is expected that the gravity partition function receives contribution from all of them, and that different ones would give the dominant contribution 
in a saddle point approximation in different regimes of the parameters. ${ }^{1}$ However, a saddle point approximation may receive leading contributions from complex on-shell actions. This fact is well-studied in the context of $\mathrm{AdS}_{5} / \mathrm{CFT}_{4}$, and an investigation of the role of the solutions contributing to the gravity integral has been recently done in [3]. Instead, we work in four Euclidean dimensions, restricting ourselves to real metric and gauge field configurations. $^{2}$ Yet, as pointed out in [6], it is possible to see a sign oscillation in some field theory partition functions. To match this in supergravity, we necessarily have to introduce an imaginary $\vartheta$ term for the Abelian gauge field, which by definition does not affect the equations of motion or the supersymmetry variations.

The holographically renormalized on-shell action of any smooth supersymmetric solution can be expressed in terms of geometric data [7]. More precisely, any supersymmetric solution admits a Killing vector $\xi$ constructed from the Killing spinor. The holographically renormalized on-shell action of a supersymmetric solution can then be expressed just in terms of contributions from the fixed point set of $\xi$, composed of isolated fixed points (nuts) and surfaces (bolts). We show that the same remains true after the introduction of the $\vartheta$ term, namely we show that

$$
\begin{aligned}
I= & \sum_{\text {nuts }} \pm\left(\frac{\pi}{2 G_{4}} \pm \frac{\mathrm{i} \vartheta}{2}\right) \frac{\left(b_{1} \pm b_{2}\right)^{2}}{4 b_{1} b_{2}} \\
& +\sum_{\text {bolts } \Sigma_{\mp}}\left(\frac{\pi}{2 G_{4}} \pm \frac{\mathrm{i} \vartheta}{2}\right) \int_{\Sigma_{ \pm}}\left(\frac{1}{2} c_{1}\left(T \Sigma_{ \pm}\right) \pm \frac{1}{4} c_{1}\left(N \Sigma_{ \pm}\right)\right) .
\end{aligned}
$$

Here \pm refer to the chirality of the Killing spinor at the fixed point; $b_{1}, b_{2}$ are the weights of the rotations generated by $\xi$ on the tangent space to the nut; $c_{1}(T \Sigma)$ and $c_{1}(N \Sigma)$ are the first Chern classes of the tangent and normal bundles to the bolt.

A crucial ingredient in the renormalization of the on-shell action in presence of the $\vartheta$ term is a finite counterterm for the $\mathrm{U}(1)$ gauge field. This is necessary in order to have consistency with the $\mathrm{SL}(2, \mathbb{Z})$ action on $3 d \mathcal{N}=2$ SCFTs [8], and with the dependence of the partition function on the background $M_{3}[9]$.

One of the far-reaching properties of the formula for the on-shell action is that we don't need the analytic form of the metric, which is generally quite difficult to find, but rather only knowledge of the topology of $Y_{4}$ and of the circle action generated by $\xi$. This poses conceptual problems, such as suggesting the existence of an underlying fixed point theorem acting on the supergravity background, especially given that this localization persists also for corrections to the two-derivative model [10-12], and it also applies to complex metrics [13]. More concretely, it provides a way of computing the on-shell action of any smooth solution, assuming its existence.

It is possible to trace the origin of the $\vartheta$ term back to eleven dimensions. Four-dimensional minimal supergravity may be derived by consistently truncating eleven-dimensional

\footnotetext{
${ }^{1}$ In fact, it is known that different methods for taking the large $N$ limit in field theory lead to matching with different supergravity solutions [2].

${ }^{2}$ This is more restrictive than the supersymmetric complex geometries that have also been studied in this context. In some cases with scalars, these have been shown to be necessary in order to match observables of four-dimensional gravity and three-dimensional field theory (see for instance [4] and [5]).
} 
supergravity on seven-dimensional internal spaces corresponding to different configurations of $M$-branes [14, 15]. Arrangements of $M 2$-branes lead to $3 d$ worldvolume theories that are typically Chern-Simons-matter theories. The dual eleven-dimensional supergravity solution has purely electric four-form flux and is an extension of the Freund-Rubin solution where the internal space is a seven-dimensional Sasaki-Einstein manifold. We show that a four-dimensional $\vartheta$ term cannot arise from this consistent truncation.

On the other hand, there is also an eleven-dimensional solutions with an $\mathrm{AdS}_{4}$ factor and magnetic four-form flux [16], which is dual to $3 d$ SCFTs obtained from M5-branes wrapping hyperbolic three-cycles $\Sigma_{3}$. The seven-dimensional internal space is an $S^{4}$ bundle over $\Sigma_{3}$, and there is a consistent truncation of eleven-dimensional supergravity on this space that leads to four-dimensional minimal supergravity. We prove that in this reduction the eleven-dimensional topological term reduces to a four-dimensional $\vartheta$ term for the gauge field, confirming the statements in [6]. Therefore, it is necessary to include a $\vartheta$ term when studying the gravity dual of the field theories obtained by wrapping $M 5$-branes on hyperbolic three-manifolds, which is a case much less studied than its previously considered counterpart [6, 17-23].

The equations of motion for the eleven-dimensional four-form are corrected by quantum effects, and the lowest-order correction has been determined [24, 25]. Reducing the corresponding term in the action, we are able to find the sub-leading correction to the expression for $\vartheta$ :

$$
\vartheta=c s\left(\Sigma_{3}\right) \frac{2 N^{3}-N}{3},
$$

where $N$ is the number of $M 5$-branes, and $\operatorname{cs}\left(\Sigma_{3}\right)$ is the Chern-Simons invariant of the hyperbolic $\Sigma_{3}$. The expression of $G_{4}$ in terms of the internal geometry can be determined at leading order in the usual way and it's proportional to the volume of $\Sigma_{3}$. More precisely, the expressions for the two four-dimensional quantities are such that the combination appearing in (1.1) takes the form

$$
\frac{\pi}{2 G_{4}}-\frac{\mathrm{i} \vartheta}{2} \longrightarrow \frac{N^{3}}{3 \pi}\left(\operatorname{Vol}\left(\Sigma_{3}\right)-\pi \mathrm{i} \operatorname{cs}\left(\Sigma_{3}\right)\right) .
$$

The bracketed quantity is a complex topological invariant of $\Sigma_{3}$ defined modulo $2 \pi^{2} \mathrm{i}$ for compact $\Sigma_{3}$, the complex hyperbolic volume. This is because Mostow's rigidity theorem guarantees that the volume is a topological invariant for hyperbolic three-manifolds.

Therefore, (1.1) tells us that the large $N$ limit of the SCFT obtained by looking at the IR limit of $N M 5$-branes wrapping $\Sigma_{3}$, denoted by $T_{N}\left[\Sigma_{3}\right]$, is proportional to a topological invariant of $\Sigma_{3}$. This is to be expected by effective field theory reasoning (the hyperbolic metric is unique, and the details are washed out in the IR), but moreover is perfectly consistent with the field-theoretic constructions of the $3 d-3 d$ correspondence [26-29]. Indeed, the latter conjectures that the partition function of $T_{N}\left[\Sigma_{3}\right]$ equals that of a $\operatorname{SL}(N, \mathbb{C})$ Chern-Simons theory on $\Sigma_{3}$, and this relation can be used to compute the large $N$ limit of the partition function of $T_{N}\left[\Sigma_{3}\right]$.

Outline. In section 2 we show how the holographically renormalized on-shell action of any smooth supersymmetric solution can be expressed in terms of data of the circle action 
generated by the Killing vector guaranteed by supersymmetry, even in presence of a $\vartheta$ term. This extends the results of [7] and shows the need of a finite counterterm for the gauge field. Then, in section 3, we consider a number of examples, both with an analytic expression for the fields and without it, including the example considered in [6].

In section 4 we consider two consistent truncations of eleven-dimensional supergravity. We briefly review the case of $Y_{4} \times S E_{7}$, showing how there cannot be a $\vartheta$ term. We then consider in detail an internal space locally $\Sigma_{3} \times S^{4}$, studying the reduction of sevendimensional maximal gauged supergravity on $\Sigma_{3}$ and finding the presence of a $\vartheta$ term. We also include some topological considerations on the global form of the eleven-dimensional solution, and the computation of the subleading term in (1.2).

Finally, in section 5 we review the large $N$ limit of the partition function of $T_{N}\left[\Sigma_{3}\right]$ on the backgrounds $M_{3}$ for which it is known, showing that they match the dual gravity computation.

In the appendices we review some conventions for the Chern-Simons terms and the construction of the complex hyperbolic volume in terms of integral of an $\operatorname{SL}(2, \mathbb{C})$ connection, and the Bott-Cattaneo formula needed for the supergravity reductions.

\section{Reduction of the supergravity action}

\subsection{Action}

We consider four-dimensional Einstein-Maxwell theory with a cosmological constant and a $\vartheta$ term. The action describes the metric $g$ and the gauge field $A$ with curvature $F=\mathrm{d} A$ with action

$$
S=-\frac{1}{16 \pi G_{4}} \int\left(R_{g}+6-F^{2}\right) \operatorname{vol}_{g}+\frac{\mathrm{i} \vartheta}{8 \pi^{2}} \int F \wedge F .
$$

The equations of motion coming from this action are

$$
\begin{aligned}
& 0=\left(\operatorname{Ric}_{g}\right)_{\mu \nu}+3 g_{\mu \nu}-2\left(F_{\mu \rho} F_{\nu}{ }^{\rho}-\frac{1}{4} F^{2} g_{\mu \nu}\right), \\
& 0=\mathrm{d} *_{g} F .
\end{aligned}
$$

With these conventions, the vacuum solution is given by $\mathrm{AdS}_{4}$ with unit radius, that is, the metric is normalized to have constant sectional curvature equal to -1 .

The action (2.1) describes the bosonic part of the minimal gauged supergravity in four dimensions. A classical solution is supersymmetric if there is a non-vanishing Dirac spinor $\epsilon$ satisfying the Killing spinor equation

$$
0=\left[\nabla-\mathrm{i} A_{\mu}+\frac{1}{2} \Gamma_{\mu}+\frac{\mathrm{i}}{4} F_{\nu \rho} \Gamma^{\nu \rho} \Gamma_{\mu}\right] \epsilon,
$$

where $\Gamma_{\mu}$ generate Cliff(4,0). As shown in [30], a spinor $\epsilon$ satisfying the Killing spinor equation cannot be everywhere chiral, so generically we have a non-zero Dirac spinor, which in four dimensions defines an identity structure. Concretely, in addition to a local orthonormal frame $\left\{\mathrm{E}^{1}, \mathrm{E}^{2}, \mathrm{E}^{3}, \mathrm{E}^{4}\right\}$, we have two functions $S$ and $\theta$

$$
S \equiv \epsilon^{\dagger} \epsilon, \quad \cos ^{2} \frac{\theta}{2} \equiv \frac{\epsilon_{+}^{\dagger} \epsilon_{+}}{S}, \quad \sin ^{2} \frac{\theta}{2} \equiv \frac{\epsilon_{-}^{\dagger} \epsilon_{-}}{S}
$$


where $\epsilon_{ \pm} \equiv \frac{1}{2}\left(\mathbb{1} \pm \Gamma_{*}\right) \epsilon$ are the chiral projections of the spinor. The structure degenerates at the points where the spinor vanishes and where it becomes chiral. ${ }^{3}$

Using the bilinears constructed from the spinor and making up the identity structure, the Killing spinor equation (2.3) can be shown to imply a number of differential equations equivalent to the equations of motion (2.2). In particular, supersymmetric solutions are naturally provided with a U(1) isometry generated by a bilinear vector field

$$
\xi^{b} \equiv-\mathrm{i} \epsilon^{\dagger} \Gamma_{(1)} \Gamma_{*} \epsilon
$$

that is also a symmetry of all the bosonic fields:

$$
\mathcal{L}_{\xi} S=\mathcal{L}_{\xi} \theta=0, \quad \mathcal{L}_{\xi} F=0, \quad \mathcal{L}_{\xi} g=0 .
$$

In fact, we have

$$
\mathrm{d} \xi^{b}=-2 S\left(\mathrm{E}^{34}+\cos \theta \mathrm{E}^{12}+*_{g} F+\cos \theta F\right)
$$

Because of the results of the supersymmetry analysis, we focus on four-dimensional space-times solving the equations (2.2) that admit a $U(1)$ symmetry generated by the vector field $\xi$ preserving both metric and gauge field. ${ }^{4}$ This assumption means that we consider manifolds $Y_{4}$ that have the structure of a circle fibration over an orbifold base $B$

$$
S^{1} \longleftrightarrow Y_{4} \backslash\left(Y_{4}\right)_{0} \longrightarrow B,
$$

where $\left(Y_{4}\right)_{0}$ is the subset of fixed points for the U(1) symmetry generated by $\xi$. Upon introducing an angle coordinate $\psi \sim \psi+\beta$ adapted to $\xi$, we may write the line element for $g$ on $Y_{4} \backslash\left(Y_{4}\right)_{0}$ as

$$
\mathrm{d} s^{2}=V(\mathrm{~d} \psi+\phi)^{2}+V^{-1} \gamma_{i j} \mathrm{~d} x^{i} \mathrm{~d} x^{j} .
$$

Here we have also introduced a local one-form $\phi$ with $\mathcal{L}_{\xi} \phi=0$ and $\left.\xi\right\lrcorner \phi=0$, a metric $\gamma$ on $B$, and the square norm of the Killing vector $V=\langle\xi, \xi\rangle_{g}$. It is a strictly positive global function on $B$, so $V^{-1}$ is well-defined, and we use it to rescale

$$
\eta \equiv V^{-1} \xi^{b} .
$$

This is a global one-form on $B$ whose local expression is $\mathrm{d} \psi+\phi$. Because of our assumptions on the symmetry of the spacetime, locally we can use gauge redundancy to write the following expression for the gauge field

$$
A=\varphi \eta+a, \quad F=\mathrm{d} \varphi \wedge \eta+\varphi \mathrm{d} \eta+f,
$$

where $a$ is a transverse one form, $\xi\lrcorner a=0, f=\mathrm{d} a$, and $\varphi$ and $a$ are both invariant under the vector field $\xi$. A constant gauge transformation along the $\mathrm{d} \psi$ direction results in

$$
\varphi \mapsto \varphi+c, \quad f \rightarrow f-c \mathrm{~d} \eta .
$$

\footnotetext{
${ }^{3}$ Since $\epsilon$ has unit charge under the U(1) gauge field, it is generically a section of a $\operatorname{spin}^{c}$ bundle over the manifold $M$, and thus the local frame is twisted. For more details, we refer to [7, 31].

${ }^{4}$ More precisely, the action is $\mathrm{U}(1)$ only if all orbits close, which we assume. If there are non-closed orbits, we would still have a torus action, and then we could approximate $\xi$ by a sequence of Killing vectors of the $\mathrm{U}(1)^{2}$. For more details, we refer to [7].
} 
Our purpose is to find an expression for the on-shell action that is exact on $B$. To do so, we reduce the action (2.1) on the circle fiber, and look at the resulting equations of motion, which correspond to the reduction of (2.2). Since the $\vartheta$ term does not modify the equations of motion, we may borrow the discussion from [7]. The result is that the bulk on-shell action may be written as an integral over the base $B$ as

$$
I_{\text {bulk }}=\int_{B} \mathrm{~d}\left[\frac{\beta}{16 \pi G_{4}} *_{\gamma}\left(\mathrm{d} \log V-\sigma H+4 V^{-1} \varphi \mathrm{d} \varphi\right)+\frac{\mathrm{i} \beta \vartheta}{8 \pi^{2}}\left(-\varphi^{2} \mathrm{~d} \eta-2 \varphi f\right)\right] .
$$

Here we have introduced the twist one-form $H \equiv *_{\gamma} \mathrm{d} \eta$ and the nut potential $\sigma$ defined by

$$
\mathrm{d} \sigma=\left(V^{2}+4 \varphi^{2} V\right) H+4 \varphi V *_{\gamma} f .
$$

The crucial step in the discussion is now the fact that the on-shell action is exact on $B$ and, provided the bracketed integrand is a global two-form on $B$, we can apply Stokes' theorem obtaining an integral on the boundary of $B$. For asymptotically locally Euclidean AdS spacetimes, this is the union of the conformal boundary and the tubular neighbourhoods surrounding the fixed points of $\xi$, where the fibration (2.8) degenerates. As we shall see, for supersymmetric solutions the regularity assumptions hold.

It is easy to make sure that the expression for the bulk on-shell action is invariant under the gauge transformation (2.12): the real part in (2.13) shifts by a multiple of the equation of motion for $\varphi$, so on-shell is gauge-invariant, and the purely imaginary part shifts by a term that vanishes using the Bianchi identity.

\subsection{Local form of supersymmetric solutions}

A careful analysis of the differential equations obtained for the bilinears constructed from the spinor satisfying (2.3) provides us with the general (local) form of the supersymmetric solution to $(2.2)[7,30]$.

We introduce on $B$ a local real coordinate $y$, a local complex coordinate $z$, and a real function $W$ so that we can write the following expressions for the metric and gauge field

$$
\begin{aligned}
\mathrm{d} s^{2} & =S^{2} \sin ^{2} \theta(\mathrm{d} \psi+\phi)^{2}+\frac{1}{y^{4} S^{2} \sin ^{2} \theta}\left(\mathrm{d} y^{2}+4 \mathrm{e}^{W} \mathrm{~d} z \mathrm{~d} \bar{z}\right), \\
A & =\left(S \cos \theta+c_{\varphi}\right)(\mathrm{d} \psi+\phi)+\frac{\mathrm{i}}{4}\left(\partial_{z} W \mathrm{~d} z-\partial_{\bar{z}} W \mathrm{~d} \bar{z}\right)
\end{aligned}
$$

where $W$ is costrained by

$$
\frac{y}{4} \partial_{y} W=1-\frac{1}{y S \sin ^{2} \theta} .
$$

These local expressions immediately give the following

$$
V=S^{2} \sin ^{2} \theta, \quad \gamma=\frac{1}{y^{4}}\left(\mathrm{~d} y^{2}+4 \mathrm{e}^{W} \mathrm{~d} z \mathrm{~d} \bar{z}\right) .
$$

We notice that the points of $Y_{4}$ where $V=0$ correspond to the points where $S=0$ or $\theta=0, \pi$, which is where the spinor vanishes or becomes chiral. Therefore, we see that $\left(Y_{4}\right)_{0}$, the fixed points of the isometry, are also the points where the supersymmetry-induced identity structure degenerates. 
Most importantly, we find expressions for $\varphi$ and the nut potential $\sigma$

$$
\varphi=S \cos \theta+c_{\varphi}, \quad \sigma=2 S \varphi+c_{\sigma}
$$

which guarantee that $\varphi$ and $\sigma$ are both global regular functions on $\left(Y_{4}\right)_{0}$, and the constants $c_{\varphi}, c_{\sigma}$ will be fixed later on. For completeness, we also write down the expressions for $\mathrm{d} \eta$

$$
\mathrm{d} \eta=2 V^{-3 / 2} *_{\gamma}\left[2 \cot \theta \mathrm{d}\left(\frac{1}{y}\right)-S \mathrm{~d} \theta\right]
$$

and two additional constraints on the functions coming from higher order differential forms constructed from the spinor

$$
\begin{gathered}
\partial_{z \bar{z}}^{2} W=-\mathrm{e}^{W}\left[\partial_{y y}^{2} W+\frac{1}{4}\left(\partial_{y} W\right)^{2}+\frac{12 \cos ^{2} \theta}{y^{4} S^{2} \sin ^{4} \theta}\right], \\
\partial_{z \bar{z}}^{2} f+\frac{\mathrm{e}^{W}}{y^{2}}\left[f\left(f^{2}+2\right)-y\left(2 \partial_{y} f+\frac{3}{2} f \partial_{y} W\right)+\right. \\
\left.+y^{2}\left(\partial_{y y}^{2} f+\frac{3}{2} \partial_{y} W \partial_{y} f+\frac{3}{2} f \partial_{y y}^{2} W+\frac{3}{4} f\left(\partial_{y} W\right)^{2}\right)\right]=0 .
\end{gathered}
$$

In the latter equation, the function $f$ is defined by

$$
f \equiv-\frac{2 \cos \theta}{y S \sin ^{2} \theta} .
$$

\subsection{On-shell action}

Thanks to the analysis of the supersymmetry equations, we have showed that the bulk onshell action (2.13) is globally exact on $B$ once we remove the points where the fibration (2.8) degenerates. More formally, we remove a tubular neighbourhood of radius $\varepsilon$ around each connected component of $\left(Y_{4}\right)_{0}$ to construct $\left(Y_{4}\right)_{\varepsilon} \subset Y_{4}$ and project to $B_{\varepsilon} \equiv\left(Y_{4} \backslash\left(Y_{4}\right)_{\varepsilon}\right) / S^{1} \subset$ $B$, which has boundaries surrounding the orbifold points (in addition to the conformal boundary). The two-form in (2.13) is globally defined on $B_{\varepsilon}$, so we may use Stokes' theorem and rewrite it as a sum of contributions from the UV conformal boundary and from the boundaries surrounding the fixed points of the isometry.

Starting from the former, for asymptotically locally Euclidean AdS solutions we interpret $1 / y$ as a radial coordinate near the conformal boundary, which we set at $\{y=$ $0\}=\partial Y_{4} \cong M_{3}$, and we assume that the various functions appearing the metric and gauge field have an analytic expansion in $y$ near the boundary. We then cut-off the spacetime to $Y_{\delta}$ where $y$ decreases up to $y=\delta>0$. The component of the boundary $\partial Y_{\delta} \equiv\{y=\delta\} \cap Y_{4} \cong M_{3}$ is still a fibered space with base $\partial B_{\delta} \equiv \partial Y_{\delta} / S^{1}$. The bulk on-shell action (2.13) gives a contribution from the UV boundary $\partial B_{\delta}$ that can be lifted to an integral over $\partial Y_{\delta}$ with the form

$$
I_{\text {bulk }}^{\mathrm{UV}}=-\int_{\partial Y_{\delta}} \eta \wedge\left[\frac{\beta}{16 \pi G_{4}} *_{\gamma}\left(\mathrm{d} \log V-\sigma H+4 V^{-1} \varphi \mathrm{d} \varphi\right)-\frac{\mathrm{i} \beta \vartheta}{8 \pi^{2}}\left(\varphi^{2} \mathrm{~d} \eta+2 \varphi f\right)\right]
$$

where the sign is due to the relation between the orientation imposed by supersymmetry and that required to apply Stokes' theorem. We should also include the Gibbons-Hawking-York 
term and the standard counterterms implementing the holographic renormalization [32, 33], which are constructed using the metric $h$ induced on the hypersurface $\partial Y_{\delta}$

$$
\begin{aligned}
I_{\mathrm{GHY}} & =-\frac{1}{8 \pi G_{4}} \int_{\partial Y_{\delta}} K \operatorname{vol}_{h}, \\
I_{\mathrm{ct}} & =\frac{1}{8 \pi G_{4}} \int_{\partial Y_{\delta}}\left(2+\frac{1}{2} R_{h}\right) \operatorname{vol}_{h} .
\end{aligned}
$$

In order to evaluate them, we impose the constraints (2.17), (2.20), (2.21) and (2.22) in the expansions of $\theta, W, S$ and $\phi$, obtaining

$$
\begin{aligned}
\theta= & \frac{\pi}{2}+y \theta_{(1)}+\frac{y^{2}}{2} \theta_{(2)}+\mathcal{O}\left(y^{3}\right) \\
W= & W_{(0)}+y W_{(1)}+\frac{y^{2}}{2}\left(-\mathrm{e}^{-W_{(0)}} \partial_{z, \bar{z}}^{2} W_{(0)}-12 \theta_{(1)}^{2}-\frac{1}{4} W_{(1)}^{2}\right)+\mathcal{O}\left(y^{3}\right), \\
S= & \frac{1}{y}+\frac{1}{4} W_{(1)}+y\left(-\frac{1}{4} \mathrm{e}^{-W_{(0)}} \partial_{z, \bar{z}} W_{(0)}-2 \theta_{(1)}^{2}\right)+\frac{y^{2}}{2}\left[\frac { 1 } { 8 } e ^ { - W _ { ( 0 ) } } \left(\partial_{z, \bar{z}}^{2} W_{(0)} W_{(1)}\right.\right. \\
& \left.\left.-2 \partial_{z, \bar{z}}^{2} W_{(1)}\right)+\frac{1}{2} W_{(1)} \theta_{(1)}^{2}-\theta_{(1)} \theta_{(2)}\right]+\mathcal{O}\left(y^{3}\right), \\
\phi= & \phi_{(0)}+y^{2} \mathrm{i}\left(\partial_{\bar{z}} \theta_{(1)} \mathrm{d} \bar{z}-\partial_{z} \theta_{(1)} \mathrm{d} z\right)+\mathcal{O}\left(y^{3}\right),
\end{aligned}
$$

where $\phi_{(0)}$ is constrained to satisfy

$$
\mathrm{d} \phi_{(0)}=4 \mathrm{ie}^{W_{(0)}} \theta_{(1)} \mathrm{d} z \wedge \mathrm{d} \bar{z} .
$$

The conclusion, as detailed in [34] is that the boundary data $W_{(0)}, \theta_{(1)}$, together with the free bulk functions $W_{(1)}, \theta_{(2)}$, determine the terms at the higher orders of the expansion.

Expanding (2.15) and (2.16) near $\partial Y_{4}$, we confirm that we have an asymptotically locally AdS space and on the conformal boundary $M_{3}$ we may pick the following representatives for the metric and gauge field

$$
\begin{aligned}
\mathrm{d} s_{(0)}^{2} & =\eta_{(0)}^{2}+4 \mathrm{e}^{W_{(0)}} \mathrm{d} z \mathrm{~d} \bar{z} \\
A_{(0)} & =-\theta_{(1)} \eta_{(0)}+\frac{\mathrm{i}}{4}\left(\partial_{z} W_{(0)} \mathrm{d} z-\partial_{\bar{z}} W_{(0)} \mathrm{d} \bar{z}\right),
\end{aligned}
$$

where $\eta_{(0)}=\mathrm{d} \psi+\phi_{(0)}, \operatorname{vol}_{2}=2 \mathrm{ie}^{W_{(0)}} \mathrm{d} z \wedge \mathrm{d} \bar{z}$ and $\square \equiv \mathrm{e}^{-W_{(0)}} \partial_{z, \bar{z}}^{2}$. This is the same geometry imposed by the presence on a three-dimensional space of two supercharges with opposite $R$-charge [35].

Indeed, this can be seen explicitly by analysing the Killing spinor equation (2.3) near the boundary, where it reduces to a conformal Killing spinor equation for a two-component spinor $\zeta$, the Killing spinor equation for three-dimensional conformal supergravity [36]. If $\zeta$ is nowhere vanishing, this is in turn equivalent to a solution of the Killing spinor equation of three-dimensional new minimal supergravity. The second "supercharge" on the three-manifold is the charge conjugate spinor $\zeta^{c} \equiv \mathcal{C}^{-1} \zeta^{*}$, which satisfies the Killing spinor equation with opposite $R$-charge. Without going into a detailed analysis of the possible backgrounds (see [37] for a review), in our case from the two supercharges we can 
construct a real Killing vector that generates a transversely holomorphic foliation. This is precisely the restriction to $M_{3}$ of $\partial_{\psi}$, and the almost contact structure one-form is $\eta_{(0)}$. By assumption, all the orbits of $\xi$ close in the bulk (see footnote 4), and so do the orbits of $\left.\xi\right|_{M_{3}}$, so the geometry of $M_{3}$ is that of a Seifert manifold, that is, a circle bundle over a two-dimensional orbifold.

The non-divergent UV contribution to the on-shell action is given by

$$
\begin{aligned}
I^{\mathrm{UV}}= & \lim _{\delta \rightarrow 0}\left[I_{\mathrm{bulk}}^{\mathrm{UV}}+I_{\mathrm{GHY}}+I_{\mathrm{ct}}\right] \\
= & \frac{1}{16 \pi^{2} G_{4}} \int_{M_{3}} \eta_{(0)} \wedge \operatorname{vol}_{2}\left[2 \pi c_{\sigma} \theta_{(1)}\right. \\
& +c_{\varphi}\left(-\mathrm{i} G_{4} \vartheta \square W_{(0)}+2 \pi \theta_{(2)}+\theta_{(1)}\left(-4 \mathrm{i} c_{\varphi} G_{4} \vartheta+3 \pi W_{(2)}\right)\right) \\
& \left.+\mathrm{i} G_{4} \vartheta\left(\theta_{(1)} \square W_{(0)}+4 \theta_{(1)}^{3}\right)\right] .
\end{aligned}
$$

There is a natural choice that simplifies the UV contribution to the on-shell action, namely $c_{\sigma}=c_{\varphi}=0$, which was called supersymmetric gauge choice in [7]

$$
I^{\mathrm{UV}}=\frac{\mathrm{i} \vartheta}{32 \pi^{2}} \int_{\partial M} \eta_{(0)} \wedge \mathrm{d} \eta_{(0)}\left(-R_{2 d}+\left(*_{2} \mathrm{~d} \eta_{(0)}\right)^{2}\right)
$$

where $R_{2 d} \equiv-\square W_{(0)}$. Notice that this is a well-defined integral over $\partial M$, and that the only UV contribution to the on-shell action comes from the $\vartheta$ term, whereas the "standard" gravitational part is determined only be the fixed points of the isometry.

We could also consider the inclusion of additional finite counterterms constructed out of the gauge fields. For standard Maxwell theory, no such counterterm is required if one fixes at the gauge field at the boundary, imposing that the dual operator in the CFT is a $\mathrm{U}(1)$ current, as we do [38]. However, in this case we also have a non-zero $\vartheta$ term, which could make the issue a bit subtler. We suggest the necessity of the following counterterm

$$
I_{\mathrm{ct}, A}=-\frac{\mathrm{i} \vartheta}{2 \pi} I_{\mathrm{CS}}\left[A_{(0)}\right]
$$

where $I_{\mathrm{CS}}[A]$ is the Chern-Simons action at level one, which for a trivial $\mathrm{U}(1)$ bundle can be written as

$$
I_{\mathrm{CS}}[A]=\frac{1}{4 \pi} \int_{M_{3}} A \wedge \mathrm{d} A .
$$

The concrete effect of this counterterm is cancelling $I^{\mathrm{UV}}$. We shall discuss this in more detail in section 2.4 .

We now move to the evaluation of the contributions to the on-shell action from the other components of the boundary of $B_{\varepsilon}$, namely the tubular neighbourhoods surrounding the fixed points of the isometry acting on $Y_{4}$. These must have even codimension, so in four dimensions we may split them into zero-dimensional nuts and two-dimensional bolts, following the terminology of [39]. As we noticed around (2.18), the fixed points of the isometry are also the loci where the identity structure degenerates. However, a more careful analysis of the bilinear equation (2.7) shows that $S$ may never vanish, unless we are in the trivial case where the isometry acts trivially everywhere. Therefore, the only 
possibility for the fixed points is that the spinor becomes chiral there. This allows us to further split the classification of the connected components of the fixed point sets according to the chirality of the supersymmetry spinor: we have nuts \pm and bolts $\Sigma_{ \pm}$depending on whether $\epsilon$ has positive/negative chirality there. Furthermore, (2.7) also gives us that at a fixed point with chirality \pm

$$
\left.S^{2}\right|_{ \pm}=\frac{1}{16}\left\langle\left(\left.\mathrm{~d} \xi^{b}\right|_{ \pm}\right)^{\mp},\left(\left.\mathrm{d} \xi^{b}\right|_{ \pm}\right)^{\mp}\right\rangle_{g}
$$

where the superscript \pm indicates the (anti-)self-dual part of the two-form.

We split the contributions of the bulk into the "standard" part, studied in [7], and the $\vartheta$ term: from (2.13) we write

$$
\begin{aligned}
I_{\text {bulk }}^{\mathrm{IR}} & =-\int_{\partial B_{\varepsilon}}\left[\frac{\beta}{16 \pi G_{4}} *_{\gamma}\left(\mathrm{d} \log V-\sigma H+4 V^{-1} \varphi \mathrm{d} \varphi\right)+\frac{\mathrm{i} \beta \vartheta}{8 \pi^{2}}\left(-\varphi^{2} \mathrm{~d} \eta-2 \varphi f\right)\right] \\
& \equiv I_{0}^{\mathrm{IR}}+I_{\vartheta}^{\mathrm{IR}}
\end{aligned}
$$

where

$$
I_{\vartheta}^{\mathrm{IR}}=\frac{\mathrm{i} \vartheta}{8 \pi^{2}} \int_{\partial M_{\varepsilon}} \eta \wedge \varphi(-\varphi \mathrm{d} \eta+2 F) .
$$

In a neighbourhood of a nut, we can write the Killing vector as the generator of the rotations on the two two-planes of $\mathbb{R}^{4} \cong \mathbb{R}^{2} \oplus \mathbb{R}^{2}$ with weights $b_{1}, b_{2}$, that is

$$
\xi=b_{1} \partial_{\varphi_{1}}+b_{2} \partial_{\varphi_{2}},
$$

where $\varphi_{1}, \varphi_{2}$ are the polar coordinates on the two copies of $\mathbb{R}^{2}$ normalised to have $2 \pi$ periodicity. Since we are assuming that the orbits of $\xi$ all close, the ratio between the two weights is rational, so we write $b_{1} / b_{2}=p / q$ with $p, q$ coprime and the period of the generic orbit is $\beta=2 \pi p / b_{1}=2 \pi q / b_{2}$. The connected component of $\partial B_{\varepsilon}$ is thus the weighted complex projective space $\mathbb{W} \mathbb{C P}_{[p,|q|]}^{1}$ and so the orbifold line bundle $\mathcal{L}$ associated to $S^{1} \hookrightarrow \partial\left(Y_{4}\right)_{\varepsilon} \rightarrow \partial B_{\varepsilon}$ has first Chern class

$$
\int_{\partial B_{\epsilon}} \mathrm{d} \eta=\beta \int_{\mathbb{W}_{[p, \mid q]]}^{1}} c_{1}(\mathcal{L})=-\frac{\beta}{p q} .
$$

In the supersymmetric gauge for $\sigma$ and $\varphi$, near a nut of type \pm we have $\left.\varphi\right|_{ \pm}= \pm\left. S\right|_{ \pm}$(as can be seen from (2.19)) and (2.38) gives

$$
\left.\varphi^{2}\right|_{\text {nut }_{ \pm}}=\frac{1}{4}\left(b_{1} \mp b_{2}\right)^{2} .
$$

Considering (2.39) term by term, we can show that

$$
\left.I_{0}^{\mathrm{IR}}\right|_{\text {nuts }}=\frac{\pi}{2 G_{4}} \sum_{\text {nuts } \mp} \pm \frac{\left(b_{1} \pm b_{2}\right)^{2}}{4 b_{1} b_{2}} .
$$

As for $I_{\vartheta}^{\mathrm{IR}}$ in (2.40), the second term in the sum is the integral over the $\partial\left(Y_{4}\right)_{\varepsilon} \cong S_{\varepsilon}^{3}$ surrounding the nut of a smooth form, so this term gives a vanishing contribution. On the other hand, the first term does contribute, and combining (2.42) and (2.43) we have

$$
\left.I_{\vartheta}^{\mathrm{IR}}\right|_{\mathrm{nuts}}=\frac{\mathrm{i} \vartheta}{2} \sum_{\text {nuts }_{\mp}} \frac{\left(b_{1} \pm b_{2}\right)^{2}}{4 b_{1} b_{2}} .
$$


Thus

$$
I_{\text {nuts }}=\sum_{\text {nuts }_{\mp}} \pm\left(\frac{\pi}{2 G_{4}} \pm \frac{\mathrm{i} \vartheta}{2}\right) \frac{\left(b_{1} \pm b_{2}\right)^{2}}{4 b_{1} b_{2}} .
$$

Given a bolt $\Sigma_{ \pm}$, we denote by $T_{\varepsilon} \cong \Sigma_{ \pm}$the connected component of $\partial B_{\epsilon}$ surrounding it. We notice that $\mathrm{d} \varphi=-\xi\lrcorner F$, which by regularity of $F$ implies that $\varphi$ is constant on the two-dimensional bolt. Therefore, we can write

$$
\lim _{\varepsilon \rightarrow 0} \int_{T_{\varepsilon}} \varphi F=\left.2 \pi \varphi\right|_{\Sigma_{ \pm}} \int_{\Sigma_{ \pm}} c_{1}(F)
$$

Analogously, we have

$$
\lim _{\varepsilon \rightarrow 0} \int_{T_{\varepsilon}} \varphi^{2} \mathrm{~d} \eta=\left.\beta \varphi^{2}\right|_{\Sigma_{ \pm}} \int_{\Sigma_{ \pm}} c_{1}\left(N \Sigma_{ \pm}\right) .
$$

To find the value of $\varphi$ at the bolt, we resort again to (2.19) and (2.38):

$$
\left.\varphi\right|_{\Sigma_{ \pm}}= \pm \frac{\pi}{\beta}
$$

Overall, (2.40) gives

$$
\left.I_{\vartheta}^{\mathrm{IR}}\right|_{\text {bolts }}=\frac{\mathrm{i} \vartheta}{2} \sum_{\text {bolts } \Sigma_{ \pm}} \int_{\Sigma_{ \pm}}\left(-\frac{1}{4} c_{1}\left(N \Sigma_{ \pm}\right) \pm c_{1}(F)\right) .
$$

This expression can be further simplified, because the existence of supersymmetry on a curved surface imposes a sort of twist. As mentioned in footnote 3, the supersymmetry spinor $\epsilon$ is generically only a section of a $\operatorname{spin}^{c}$ bundle that is twisted by the $\mathrm{U}(1)$ gauge field $A$. Near a bolt, the spinor ${ }^{c}$ becomes chiral but does not vanish, so there is a non-zero section of a line bundle over the bolt, meaning that said bundle must be trivial. This constraint imposes a relation between the restriction of the gauge bundle over $\Sigma_{ \pm}$and the tangent and normal bundle to the surface [7]

$$
\int_{\Sigma_{ \pm}} c_{1}(F)=\int_{\Sigma_{ \pm}} \frac{ \pm c_{1}\left(N \Sigma_{ \pm}\right)-c_{1}\left(T \Sigma_{ \pm}\right)}{2}
$$

Inserting the latter result in the expression (2.50) leads to the result

$$
\left.I_{\vartheta}^{\mathrm{IR}}\right|_{\text {bolts }}=\frac{\mathrm{i} \vartheta}{2} \sum_{\text {bolts } \Sigma_{ \pm}} \int_{\Sigma_{ \pm}}\left(\frac{1}{4} c_{1}\left(N \Sigma_{ \pm}\right) \mp \frac{1}{2} c_{1}\left(T \Sigma_{ \pm}\right)\right)
$$

and a similar analysis for $I_{0}^{\mathrm{IR}}$ gives

$$
\left.I_{0}^{\mathrm{IR}}\right|_{\text {bolts }}=\frac{\pi}{2 G_{4}} \sum_{\text {bolts } \Sigma_{ \pm}} \mp \int_{\Sigma_{ \pm}}\left(\frac{1}{4} c_{1}\left(N \Sigma_{ \pm}\right) \mp \frac{1}{2} c_{1}\left(T \Sigma_{ \pm}\right)\right)
$$

whence

$$
I_{\text {bolts }}=\sum_{\text {bolts } \Sigma_{ \pm}}\left(\frac{\pi}{2 G_{4}} \mp \frac{\mathrm{i} \vartheta}{2}\right) \int_{\Sigma_{ \pm}}\left(\frac{1}{2} c_{1}\left(T \Sigma_{ \pm}\right) \mp \frac{1}{4} c_{1}\left(N \Sigma_{ \pm}\right)\right) .
$$

Summing the contributions from all the fixed points of the isometry, we find that the "infrared" contribution to the on-shell action with $\vartheta$ term is

$$
\begin{aligned}
I^{\mathrm{IR}}= & \sum_{\text {nuts }} \pm\left(\frac{\pi}{2 G_{4}} \pm \frac{\mathrm{i} \vartheta}{2}\right) \frac{\left(b_{1} \pm b_{2}\right)^{2}}{4 b_{1} b_{2}} \\
& +\sum_{\text {bolts } \Sigma_{\mp}}\left(\frac{\pi}{2 G_{4}} \pm \frac{\mathrm{i} \vartheta}{2}\right) \int_{\Sigma_{ \pm}}\left(\frac{1}{2} c_{1}\left(T \Sigma_{ \pm}\right) \pm \frac{1}{4} c_{1}\left(N \Sigma_{ \pm}\right)\right) .
\end{aligned}
$$




\subsection{Dependence on the boundary and periodicity of $\vartheta$}

Having found an expression for the on-shell action of any smooth supergravity solution, it seems redundant to investigate its dependence on boundary data. Nonetheless, it is an additional check on our expression. We compute the one-point function of the holographic stress-energy tensor and the $\mathrm{U}(1)_{R}$ current: the holographic stress-energy tensor has the same form as in [34], whereas the $\mathrm{U}(1)_{R}$ current receives an additional contribution from the $\vartheta$ term

$$
j_{\vartheta}=\frac{\mathrm{i} \vartheta}{4 \pi^{2}}\left[\left(2 \theta_{(1)}^{2}+\frac{1}{4} \square W_{(0)}\right) \eta_{(0)}-*_{2} \mathrm{~d} \theta_{(1)}\right] .
$$

Under a change in the boundary geometry and $\mathrm{U}(1)_{R}$ gauge field given by $W_{(0)} \rightarrow W_{(0)}+$ $\delta W_{(0)}, \phi_{(0)} \rightarrow \phi_{(0)}+\delta \phi_{(0)}$, where the variations are a basic function and one-form on $\partial Y_{4}$, respectively, we find that the variation of the holographically renormalized on-shell action vanishes [34], apart from the $\vartheta$ term, which gives

$$
\delta I=\frac{\mathrm{i} \vartheta}{16 \pi^{2}} \int_{\partial M} \eta_{(0)} \wedge \delta\left(\theta_{(1)} \square W_{(0)} \operatorname{vol}_{2}+4 \theta_{(1)}^{3} \operatorname{vol}_{2}\right) .
$$

Looking at this, it is clear that the non-zero variation is entirely due to (2.35).

There is an expectation stemming from field theory that the partition function of a three-dimensional $\mathcal{N}=2$ field theory on a background preserving at least two supercharges (as the one we find at the boundary of $Y_{4}$ ) should only depend on the background geometry via the choice of transversely holomorphic foliation [9]. Because of the AdS/CFT correspondence, the renormalized on-shell action of the dual gravity solution should have the same dependence on the boundary data. As proved in [34], this holds for the canonical action of four-dimensional minimal supergravity. However, the computation just presented shows that this does not hold true when we include a $\vartheta$ term, since the specific variation we considered preserves the transversely holomorphic foliation and yet $\delta I \neq 0$. This is a first piece of evidence in favour of the finite counterterm (2.36), which cancels $I^{\mathrm{UV}}$ thus removing $\delta I .^{5}$

Additional evidence comes from a more careful thought on the periodicity of the $\vartheta$ angle. Abelian gauge theories in four dimensions on a spin manifold enjoy a $\operatorname{SL}(2, \mathbb{Z})$ duality group [43]. The $T$ generator corresponds to the shift $\vartheta \rightarrow \vartheta+2 \pi$, whereas the $S$ generator, which does not commute with $T$, is the exchange of electric and magnetic fields.

If $Y_{4}$ is closed and spin, then $\int_{Y_{4}} F \wedge F \in 8 \pi^{2} \mathbb{Z}$, so the theory for which (2.1) is the semiclassical effective description is invariant under $\vartheta \rightarrow \vartheta+2 \pi$, since $\mathrm{e}^{-S}$ would be unchanged. The main focus of the paper, though, are asymptotically locally AdS spaces, more precisely cutoff at a distance $\delta$ from the conformal boundary. On a space $Y_{4}$ with a boundary $M_{3}$, we extend the line bundle $L$ on $Y_{4}$ to a line bundle $L_{(0)}$ on $M_{3}$. Then, $\int_{Y_{4}} F \wedge F$ fails to be a multiple of $8 \pi^{2}$ by a boundary Chern-Simons term at level one (2.37). That is, after shifting $\vartheta \rightarrow \vartheta+2 \pi$, we find

$$
S \rightarrow S+2 \pi \mathrm{i} \ell+\mathrm{i} I_{\mathrm{CS}}\left[A_{(0)}\right], \quad \ell \in \mathbb{Z} .
$$

\footnotetext{
${ }^{5}$ The necessity of finite counterterms in order to be preserve boundary supersymmetry is well-known in cases with scalars and additional supersymmetry, e.g. [5, 40-42].
} 
This shift leads to an action on the partition function of the boundary theory

$$
Z_{\mathrm{SCFT}}\left[A_{(0)} ; M_{3}\right]=\mathrm{e}^{-S\left[A ; Y_{4}\right]} \rightarrow Z_{\mathrm{SCFT}}\left[A_{(0)} ; M_{3}\right] \mathrm{e}^{-\mathrm{i} I_{\mathrm{CS}}\left[A_{(0)}\right]} .
$$

This is expected from field theory: three-dimensional conformal field theories with a U(1) global symmetry are acted upon by a $\mathrm{SL}(2, \mathbb{Z})$ group. More precisely, after coupling the $\mathrm{U}(1)$ current to a background gauge field $A_{(0)}$, one finds that the $T$ generator of $\operatorname{SL}(2, \mathbb{Z})$ acts on the generating functional of the correlation functions of the $\mathrm{U}(1)$ current by shifting it by a level one Chern-Simons term for the background gauge field. This is precisely our setup, since we are describing the universal sector of three-dimensional SCFTs with $\mathcal{N}=2$, which always have a $\mathrm{U}(1)_{R}$ global $R$-symmetry. The $\mathrm{SL}(2, \mathbb{Z})$ group acting on $3 d$ CFTs becomes in the bulk the duality group of four-dimensional U(1) gauge theory on $\mathrm{AlAdS}_{4}$.

Notice that the boundary $\mathrm{SL}(2, \mathbb{Z})$ group is not a duality, since its action generically modifies the $3 d$ CFT. Correspondingly, the $\mathrm{SL}(2, \mathbb{Z})$ action on the Einstein-Maxwell theory on anti-de Sitter space is to be interpreted as a choice of boundary conditions for the gauge field $[8,44-47]$. On the other hand, the introduction of the counterterm (2.36) fixes the choice of boundary conditions by removing the additional boundary Chern-Simons term

$$
S+I_{\mathrm{ct}, A} \rightarrow S+I_{\mathrm{ct}, A}+2 \pi \mathrm{i} \ell, \quad \ell \in \mathbb{Z} .
$$

This guarantees that the bulk theory is invariant under $\vartheta \rightarrow \vartheta+2 \pi$.

As described after (2.33), the boundary superconformal field theory is formulated on $M_{3}$ by coupling to the multiplet of a background three-dimensional conformal supergravity, which includes $g_{(0)}$ and $A_{(0)}$. The latter is the background gauge field coupling to the $R$ symmetry current. A Chern-Simons term for $A_{(0)}$ is related by $\mathcal{N}=2$ supersymmetry to the gravitational Chern-Simons term [48], so their levels are related. The fractional part of this level is a physical quantity of the SCFT: it cannot be removed by a local gauge-invariant counterterm, and it appears as a conformally-invariant contact term in the two-point function of the stress-energy tensor [49]. This fractional part is captured by the counterterm (2.36), being $\vartheta / 2 \pi \bmod 1$, and consistently the shift $\vartheta \rightarrow \vartheta+2 \pi$ does not modify it.

\section{$3 \quad$ Examples}

\section{$3.1 \quad \mathrm{AdS}_{4}$}

The simplest non-trivial solution we can consider is $\mathrm{AdS}_{4}$ with an instanton [50, 51]. The metric and gauge field are

$$
\begin{aligned}
\mathrm{d} s^{2} & =\frac{\mathrm{d} r^{2}}{r^{2}+1}+r^{2}\left(\mathrm{~d} \vartheta^{2}+\cos ^{2} \vartheta \mathrm{d} \varphi_{1}^{2}+\sin ^{2} \vartheta \mathrm{d} \varphi_{2}^{2}\right) \\
A & =-\frac{\left(b_{1}+b_{2} \sqrt{r^{2}+1}\right) \mathrm{d} \varphi_{1}+\left(b_{2}+b_{1} \sqrt{r^{2}+1}\right) \mathrm{d} \varphi_{2}}{2 \sqrt{\left(b_{2}+b_{1} \sqrt{r^{2}+1}\right)^{2} \cos ^{2} \vartheta+\left(b_{1}+b_{2} \sqrt{r^{2}+1}\right)^{2} \sin ^{2} \vartheta}},
\end{aligned}
$$

where $\vartheta \in\left[0, \frac{\pi}{2}\right], \varphi_{1}, \varphi_{2} \in[0,2 \pi]$, and there is a $\mathrm{U}(1) \times \mathrm{U}(1)$ Killing vector field constructed from the Killing spinor

$$
\xi=b_{1} \partial_{\varphi_{1}}+b_{2} \partial_{\varphi_{2}}
$$


This solution is only regular provided $b_{1} / b_{2} \equiv b^{2}>0$ and $b_{1} / b_{2}=-1$. In the cases $b_{1} / b_{2}= \pm 1$ it reduces to the trivial instanton.

The norm of the Killing vector is $V=r^{2}\left(b_{1} \cos ^{2} \phi_{1}+b_{2} \sin ^{2} \phi_{2}\right)$, which shows that at $r=0$ there is a nut. In fact, a more careful analysis leads to the conclusion that for $b_{1} / b_{2}=b^{2}$ we have a nut - and for $b_{1} / b_{2}=-1$ we have a nut + . In the two cases applying (2.55) leads to

$$
\frac{b_{1}}{b_{2}}=-1: \quad I^{\mathrm{IR}}=\frac{\pi}{2 G_{4}}-\frac{\mathrm{i} \vartheta}{2}, \quad \frac{b_{1}}{b_{2}}=b^{2}: \quad I^{\mathrm{IR}}=\frac{1}{4}\left(\frac{\pi}{2 G_{4}}+\frac{\mathrm{i} \vartheta}{2}\right)\left(b+\frac{1}{b}\right)^{2} .
$$

Instead, applying (2.35) gives

$$
I^{\mathrm{UV}}=-\frac{\mathrm{i} \vartheta}{2} \frac{b_{1}^{2}+b_{2}^{2}}{2 b_{1} b_{2}}
$$

The smooth solution with $b_{1} / b_{2}=b^{2}$ has another interpretation: $\mathrm{AdS}_{4}$ (with the nontrivial instanton) can be foliated in a non-standard way to find a conformal boundary that is isometric to a squashed $S^{3}$ [50]. More concretely, we perform the change of coordinates

$$
\begin{aligned}
r^{2} & =\left(\frac{q^{2}}{b^{2}}-1\right) \cos ^{2} \psi+\left(q^{2} b^{2}-1\right) \sin ^{2} \psi, \\
\cos ^{2} \vartheta & =\frac{\left(\frac{q^{2}}{b^{2}}-1\right) \cos ^{2} \psi}{\left(\frac{q^{2}}{b^{2}}-1\right) \cos ^{2} \psi+\left(q^{2} b^{2}-1\right) \sin ^{2} \psi},
\end{aligned}
$$

obtaining

$$
\begin{aligned}
\mathrm{d} s^{2}= & \frac{q^{2}-f(\psi)^{-2}}{\left(q^{2}-b^{2}\right)\left(q^{2}-b^{-2}\right)} \mathrm{d} q^{2}+\left(q^{2} f(\psi)^{2}-1\right) \mathrm{d} \psi^{2} \\
& +\left(\frac{q^{2}}{b^{2}}-1\right) \cos ^{2} \psi \mathrm{d} \varphi_{1}^{2}+\left(q^{2} b^{2}-1\right) \sin ^{2} \psi \mathrm{d} \varphi_{2}^{2}
\end{aligned}
$$

with

$$
f(\psi)^{2} \equiv \frac{1}{b^{-2} \cos ^{2} \psi+b^{2} \sin ^{2} \psi} .
$$

The metric (3.6) is again $\mathrm{AdS}_{4}$, but now the metric at the conformal boundary at $q \rightarrow \infty$ is

$$
\mathrm{d} s_{3}^{2}=f(\psi)^{2} \mathrm{~d} \psi^{2}+\frac{1}{b^{2}} \cos ^{2} \psi \mathrm{d} \varphi_{1}^{2}+b^{2} \sin ^{2} \psi \mathrm{d} \varphi_{2}^{2} .
$$

This is the metric on the squashed three-sphere preserving $\mathrm{U}(1) \times \mathrm{U}(1)$ symmetry and two supercharges studied in [52]. To further confirm the smoothness of the slicings, notice that (3.8) is related to the metric on the round sphere by a Weyl transformation: upon substituting

$$
\cos ^{2} \vartheta=\frac{b^{-2} \cos ^{2} \psi}{b^{-2} \cos ^{2} \psi+b^{2} \sin ^{2} \psi}
$$

we find that

$$
\mathrm{d} \vartheta^{2}+\cos ^{2} \vartheta \mathrm{d} \varphi_{1}^{2}+\sin ^{2} \vartheta \mathrm{d} \varphi_{2}^{2}=f(\psi)^{2}\left(f(\psi)^{2} \mathrm{~d} \psi^{2}+b^{-2} \cos ^{2} \psi \mathrm{d} \varphi_{1}^{2}+b^{2} \sin ^{2} \psi \mathrm{d} \varphi_{2}^{2}\right) .
$$


In fact, the form of $f(\psi)$ is not relevant for the large $N$ limit of the localization computation of the dual field theory, as showed in [53]. It is sufficient that it guarantees smoothness of the metric, that is, it has the behaviour

$$
\lim _{\psi \rightarrow 0} f(\psi)=b, \quad \lim _{\psi \rightarrow \frac{\pi}{2}} f(\psi)=\frac{1}{b} .
$$

Consistently, we shall see that this holds for $I^{\mathrm{UV}}$ as well. Given the metric (3.8) and the Killing vector $\xi=b \partial_{\varphi_{1}}+b^{-1} \partial_{\varphi_{2}}$ (which is (3.2) when $b_{2}=b^{-1}$ ), then $\mathrm{d} \eta=2 \operatorname{vol}_{2} / f(\psi)$, and $R_{2 d}=4\left(2 f(\psi)+f^{\prime}(\psi) \cot 2 \psi\right) / f(\psi)^{3}$ so that (2.35) leads to

$$
I^{\mathrm{UV}}=\frac{\mathrm{i} \vartheta}{2} \int_{0}^{\pi / 2} \frac{\mathrm{d}}{\mathrm{d} \psi}\left(\frac{\cos 2 \psi}{2 f(\psi)^{2}}\right) \mathrm{d} \psi=-\frac{\mathrm{i} \vartheta}{2} \frac{b^{2}+b^{-2}}{2},
$$

consistently with (3.4) and independently on the details of $f(\psi)$.

\subsection{Supersymmetric black holes}

Another simple and yet interesting solution that we consider are the supersymmetric black holes obtained by Wick-rotation of the dyonic static solutions of [54-56]. These are related to the solutions considered in [6]. They preserve $1 / 4$ of the supersymmetry and have the topology $\mathbb{R}^{2} \times \Sigma_{g}$ for $g>1$. The local form of the metric and gauge field is

$$
\begin{aligned}
\mathrm{d} s^{2} & =V(r) \mathrm{d} \tau^{2}+\frac{\mathrm{d} r^{2}}{V(r)}+r^{2} \mathrm{~d} s^{2}\left(\Sigma_{g}\right), \quad V=-1+\frac{\frac{1}{4}-Q^{2}}{r^{2}}+r^{2}, \\
F & =\frac{Q}{r^{2}} \mathrm{~d} \tau \wedge \mathrm{d} r+\frac{1}{2} \operatorname{vol}\left(\Sigma_{g}\right)
\end{aligned}
$$

If $Q \neq 0$, the solution is regular provided $r>r_{0}$ with $r_{0}=\sqrt{\frac{1}{2}+|Q|}$ and $\tau \sim \tau+\frac{4 \pi}{\left|V^{\prime}\left(r_{0}\right)\right|}$. If $Q=0$, instead, the metric develops an infinite throat near $r=1 / \sqrt{2}$.

The Killing vector $\xi$ constructed from the Killing spinor is $\xi=\partial_{\tau}$. It has a bolt at $r=r_{0}$ if $Q \neq 0$ that goes to infinite distance as $Q \rightarrow 0$. Provided $Q \neq 0$, we have a smooth metric with a bolt \pm where the chirality of the spinor at the bolt is equal to $\operatorname{sgn}(Q)$. Since the fibration is trivial, equation (2.35) vanishes and the only contribution comes from the IR $(2.55)$

$$
I=\left(\frac{\pi}{2 G_{4}}-\operatorname{sgn}(Q) \frac{\mathrm{i} \vartheta}{2}\right)(1-g) .
$$

The only dependence on $Q$ in the action is in the $\vartheta$ term, and we will obtain different results depending on whether we take $Q \rightarrow 0^{+}$or $Q \rightarrow 0^{-}$. The same result has been obtained in [6] using a different regularization of the extremal metric with $Q=0$ breaking supersymmetry.

\section{$3.3 \quad \frac{1}{4}$-BPS solutions}

There are also a number of solutions preserving two real supercharges but with a non-trivial fibration. Here we consider those constructed in [2,31] and further analysed in [34, 51]. 
They all have the form

$$
\begin{aligned}
\mathrm{d} s^{2} & =\frac{r^{2}-s^{2}}{\Omega(r)} \mathrm{d} r^{2}+\left(r^{2}-s^{2}\right)\left(\sigma_{1}^{2}+\sigma_{2}^{2}\right)+4 s^{2} \frac{\Omega(r)}{r^{2}-s^{2}} \sigma_{3}^{2}, \\
A & =\frac{P\left(r^{2}+s^{2}\right)-2 Q r s}{\left(r^{2}-s^{2}\right)} \sigma_{3}
\end{aligned}
$$

for different choices of $\Omega(r), P, Q$ and the forms $\sigma_{i}$.

There is a Einstein Taub-NUT-AdS solution on $\mathbb{R}^{4} / \mathbb{Z}_{p}$ (with an instanton) for the choices

$$
\Omega(r)=(r-s)^{2}[1+(r-s)(r+3 s)], \quad Q=P=-\frac{4 s^{2}-1}{2},
$$

and the $\sigma_{i}$ are the left-invariant one-forms on $S^{3}$

$$
\sigma_{1}+\mathrm{i} \sigma_{2}=\mathrm{e}^{-\mathrm{i} \tau}(\mathrm{d} \vartheta+\mathrm{i} \sin \vartheta \mathrm{d} \phi), \quad \sigma_{3}=\mathrm{d} \tau+\cos \vartheta \mathrm{d} \phi .
$$

This is a self-dual metric, in the sense that both the gauge field and the Weyl tensor are self-dual. The Killing vector constructed from the Killing spinor is $\xi=\partial_{\tau}$, so $\eta=\sigma_{3}$. We use the gauge freedom in (2.12) to find that the function $\varphi$ satisfying the supersymmetric gauge choice is

$$
\varphi=-\frac{\left(4 s^{2}-1\right)(r-s)}{2(r+s)}-\frac{1}{2} .
$$

The conformal boundary is a squashed $S^{3} / \mathbb{Z}_{p}$ with metric

$$
\mathrm{d} s_{3}^{2}=\sigma_{1}^{2}+\sigma_{2}^{2}+4 s^{2} \sigma_{3}^{2} .
$$

This squashed sphere preserves $\mathrm{SU}(2) \times \mathrm{U}(1)$ and supports two real supercharges [52]. In order to apply (2.35), we let $\eta_{(0)}=2 s \sigma_{3}$, from which $R_{2 d}=2$. Thus

$$
I^{\mathrm{UV}}=\frac{\mathrm{i} \vartheta}{2} \frac{16 s^{4}-8 s^{2}}{p} .
$$

Given the form of $\Omega$ and $Q, P$, we find that $\xi$ has a nut at $r=s$, corresponding to the origin of the $\mathbb{R}^{4}$ where we should take $\tau \sim \tau+\frac{4 \pi}{p}$ for regularity. In our terminology, the origin is a nut_ with $b_{1}=b_{2}$, so $(2.55)$ gives

$$
I^{\mathrm{IR}}=\frac{\pi}{2 G_{4}}+\frac{\mathrm{i} \vartheta}{2} .
$$

The overall action with $\vartheta$ term, which is consistent with [31], is the sum of the two contributions.

In addition to the self-dual Taub-NUT-AdS solution with topology $\mathbb{R}^{4} / \mathbb{Z}_{p}$, there is also a family of solutions with topology $\mathcal{O}(-p) \rightarrow \Sigma_{g}$ for any $\Sigma_{g}$ with metric with constant sectional curvature $\kappa$. In this case the metric and gauge field still have the form (3.15), but now

$$
\begin{aligned}
& \Omega=\left(r^{2}-s^{2}\right)^{2}+\left(\kappa-4 s^{2}\right)\left(r^{2}-s^{2}\right)-4 s Q r+\frac{1}{4}\left(4 s^{2}-\kappa\right)^{2}-Q^{2}, \\
& P=-\frac{4 s^{2}-\kappa}{2} .
\end{aligned}
$$


and for the one-forms defining the metric we still use (3.17) for $\Sigma_{0} \cong S^{2}$, but otherwise we locally define

$$
\begin{aligned}
& \Sigma_{1} \cong T^{2} \quad \sigma_{1}+\mathrm{i} \sigma_{2}=\mathrm{e}^{\mathrm{i} \tau}(\mathrm{d} \phi-\mathrm{i} \mathrm{d} \vartheta), \quad \sigma_{3}=\mathrm{d} \tau-\vartheta \mathrm{d} \phi, \\
& \Sigma_{g} g>1 \quad \sigma_{1}+\mathrm{i} \sigma_{2}=\mathrm{e}^{\mathrm{i} \tau}(\sinh \vartheta \mathrm{d} \phi-\mathrm{i} \mathrm{d} \vartheta), \quad \sigma_{3}=\mathrm{d} \tau-\cosh \vartheta \mathrm{d} \phi .
\end{aligned}
$$

Again, the Killing vector constructed from supersymmetry is $\xi=\partial_{\tau}$, which generates the fibration and whose norm vanishes at the zeroes of $\Omega$. If $Q= \pm \frac{1}{2} \sqrt{4 s^{2}-\kappa}$, then we can only have $\kappa=1$ and we obtain the Taub-NUT-AdS solution considered earlier, otherwise smoothness of the solution at $r_{0}$, the largest root of $\Omega$, fixes $Q$ by imposing

$$
\frac{r_{0}^{2}-s^{2}}{\left|\Omega^{\prime}\left(r_{0}\right)\right|}=\frac{2 s}{\mathbf{p}}, \quad \text { with } \mathbf{p}=\frac{p}{|g-1|} \text { for } g \neq 1, \text { and } \mathbf{p}=p \text { for } g=1 \text {. }
$$

Analogously, the period of $\tau$ is fixed to be

$$
\tau \sim \tau+\frac{4 \pi}{\mathbf{p}}
$$

There are two branches of solutions, whose existence depends on the intricate relation between the deformation parameter $s$ and the integer $p$. We refer to these branches as "Bolt ${ }_{ \pm}$", consistently with $[2,31]$. For both branches the Killing vector $\xi$ has a bolt at $r=r_{0}$ that is diffeomorphic to $\Sigma_{g}$, the basis of the fibration. Studying the form of the functions defined in section 2.2 near the bolt shows that for the solution Bolt \pm we have a bolt $\mp$ in our language: the chirality of the spinor is opposite to the sign identifying the branch of solutions. Thus we can apply (2.55) and find in the IR

$$
I^{\mathrm{IR}}\left[\text { Bolt }_{ \pm}\right]=\left(\frac{\pi}{2 G_{4}} \pm \frac{\mathrm{i} \vartheta}{2}\right)\left(1-g \mp \frac{p}{4}\right),
$$

since $T \Sigma_{g} \cong \mathcal{O}(2-2 g)$ and $N \Sigma_{g} \cong \mathcal{O}(-p)$.

The conformal boundary is the manifold $\mathcal{M}_{g, p}$, a $S^{1}$ bundle of degree $p$ over the Riemann surface $\Sigma_{g}$, which includes the $S^{3} / \mathbb{Z}_{p}$ bounding the self-dual Taub-NUT-AdS solution. Indeed, the structure of the UV contribution is not very different from (3.20): again $\eta_{(0)}=2 s \sigma_{3}$ and $\mathrm{d} \eta_{(0)}=2 s \operatorname{vol}\left(\Sigma_{g}\right)$. The metric on the Riemann surface is normalized so that $R_{2 d}=2 \kappa$ and $\operatorname{vol}\left(\Sigma_{g}\right)$ integrates to $\frac{4 \pi p}{\mathbf{p}}$. Therefore $(2.35)$ gives

$$
I^{\mathrm{UV}}=\frac{\mathrm{i} \vartheta}{2} \frac{\left(16 s^{4}-8 s^{2} \kappa\right) p}{\mathbf{p}^{2}}=\left\{\begin{array}{ll}
\frac{\mathrm{i} \vartheta}{2} \frac{\left(16 s^{4}-8 s^{2} \kappa\right)(g-1)^{2}}{p} & g \neq 1 \\
\frac{\mathrm{i} \vartheta}{2} \frac{16 s^{4}}{p} & g=1
\end{array} .\right.
$$

Notice that this reduces to (3.20) for $g=0, \kappa=1$. Moreover, for the trivial fibration $p=0$ $I^{\mathrm{UV}}=0$ and (3.26) corresponds to (3.14) provided we identify the sign of the branch of the bolt solution with $-\operatorname{sgn}(Q)$.

\section{$3.4 \quad \frac{1}{2}$-BPS solutions}

There are also known solutions preserving four real supercharges, such as those constructed in [31]. The local form of the metric is again (3.15), but now the forms $\sigma_{i}$ are always the left-invariant one-forms on $S^{3}(3.17)$. 
The simplest case is again the Einstein Taub-NUT-AdS solution on $\mathbb{R}^{4} / \mathbb{Z}_{p}[51,57]$, but now with a different instanton:

$$
\Omega(r)=(r-s)^{2}[1+(r-s)(r+3 s)], \quad Q=P=-s \sqrt{4 s^{2}-1} .
$$

The Killing vector determined by supersymmetry depends on two numbers (p, q) $\in \mathbb{C}^{2} \backslash\{0\}$. As showed in [7], the final result does not depend on the choice of $p, q$ provided one of the two vanishes, so we choose $p=1, q=0$. Then

$$
\xi=-2\left[\left(2 s+\sqrt{4 s^{2}-1}\right) \partial_{\phi}+\left(\frac{1}{2 s}-2 s-\sqrt{4 s^{2}-1}\right) \partial_{\tau}\right]
$$

In contrast with the $\frac{1}{4}$-BPS solutions considered earlier, we see that $\xi \neq \partial_{\tau}$, so the Killing vector induced by supersymmetry is not the one generating the fibration of the solution. Nonetheless, one can show that $\xi$ has a nut at the origin of $\mathbb{R}^{4}$ at $r=s$ : in our language it is a nut + with

$$
b_{1}=-\frac{1}{2 s}, \quad b_{2}=-\frac{1}{2 s}+4 s+2 \sqrt{4 s^{2}-1} .
$$

Thus (2.55) gives

$$
I^{\mathrm{IR}}=\left(\frac{\pi}{2 G_{4}}-\frac{\mathrm{i} \vartheta}{2}\right) 4 s^{2} .
$$

Despite the fact that the conformal boundary of these solutions is geometrically $S^{3} / \mathbb{Z}_{p} \cong$ $\mathcal{M}_{0, p}$ as for the solutions considered in the previous section, the contribution to the action is different, because the Killing vector $\xi$ is different. This is due to the fact that these solutions, and hence their boundary, preserves four supercharges instead of two. In this case, application of (2.35) leads to

$$
I^{\mathrm{UV}}=\frac{\mathrm{i} \vartheta}{2} \frac{16 s^{4}}{p}
$$

and so

$$
I_{\vartheta}=\frac{\mathrm{i} \vartheta}{2} 4 s^{2}\left(\frac{4 s^{2}}{p}-1\right)
$$

which is consistent with [31].

As for the $\frac{1}{4}$-BPS solutions, there is a family of non-self-dual solutions with topology $\mathcal{O}(-p) \rightarrow S^{2}$ with

$$
\begin{aligned}
& \Omega=\left(r^{2}-s^{2}\right)^{2}+\left(1-4 s^{2}\right)\left(r^{2}-s^{2}\right)-2 Q \sqrt{4 s^{2}-1} r+s^{2}\left(4 s^{2}-1\right)-Q^{2}, \\
& P=-s \sqrt{4 s^{2}-1} .
\end{aligned}
$$

The parameter $Q$ is fixed by regularity of the metric at $r_{0}$, the largest root of $\Omega$, and this condition identifies two branches of solutions. Since the form of metric is again (3.15), the locus $\left\{r=r_{0}\right\}$ is a $S^{2}$ bolt for the Killing vector $\partial_{\tau}$ for the $\frac{1}{2}$-BPS solutions as well, therefore in the literature the two branches of solutions are again called "Bolt \pm ". The difference with the previous case is that now $\xi \neq \partial_{\tau}$, and $\xi$ doesn't have a bolt. In fact, $\xi$ 
vanishes only at the poles of the $S^{2}$. More precisely, for the Bolt \pm solution, the north pole is a nut \pm with weights

$$
b_{1}=-4 s-2 \sqrt{4 s^{2}-1}, \quad b_{2}=\frac{p}{2 s},
$$

and the south pole is a nut $\mp$ with weights

$$
b_{1}=4 s+2 \sqrt{4 s^{2}-1}, \quad b_{2}=\frac{p}{2}\left(\frac{1}{s}-8 s-4 \sqrt{4 s^{2}-1}\right) .
$$

Applying (2.55) then gives

$$
I^{\mathrm{IR}}\left[\mathrm{Bolt}_{ \pm}\right]=\frac{\pi}{2 G_{4}}\left[1 \pm \frac{2 \sqrt{4 s^{2}-1}}{s p}\left(s^{2}-\frac{p^{2}}{16}\right)\right]+\frac{\mathrm{i} \vartheta}{2}\left(-\frac{p}{4}-\frac{4 s^{2}}{p}\right)
$$

Since the conformal boundary data are the same for self-dual and non-self-dual solutions, the contribution from the conformal boundary is again (3.33).

\subsection{Other topologies}

For the examples considered so far we know an analytic expression for the metric and gauge field. However, this is generically hard to find. The formulae found here and in [7] only require knowledge of topological data at the fixed points of the $\mathrm{U}(1)$ action, without the details of the fields in the bulk.

For instance, suppose that $Y_{4}=\mathcal{O}(-p) \rightarrow S^{2}$, assume the existence of a supersymmetric solution on $Y_{4}$, and that the isometry generated by $\xi$ is contained in the natural $\mathrm{U}(1)^{2}$ acting on fiber and base. Then one can use simple toric geometry to find an expression for the on-shell action contribution (2.55) by straightforward generalization of [7]. In particular, a solution will have a Killing vector $\xi$ with components $\left(a_{1}, a_{2}\right)$ on the basis of the torus action, and this Killing vector will have two nuts with chiralities $\kappa_{1}, \kappa_{2}$ and weights $b_{i}^{(1)}, b_{i}^{(2)}$. The IR contribution to the on-shell action is

$$
\begin{aligned}
I^{\mathrm{IR}} & =\sum_{i=1}^{2}-\kappa_{i}\left(\frac{\pi}{2 G_{4}}-\kappa_{i} \frac{\mathrm{i} \vartheta}{2}\right) \frac{\left(b_{1}^{(i)}-\kappa_{i} b_{2}^{(i)}\right)^{2}}{4 b_{1}^{(i)} b_{2}^{(i)}} \\
& =\frac{1}{4 a_{1}\left(a_{1}+a_{2}\right)\left(a_{1}(p-1)-a_{2}\right)}\left(\mathcal{Q}_{p}\left(\kappa_{1}, \kappa_{2} ; a_{1}, a_{2}\right) \frac{\pi}{2 G_{4}}-\mathcal{R}_{p}\left(\kappa_{1}, \kappa_{2} ; a_{1}, a_{2}\right) \frac{\mathrm{i} \vartheta}{2}\right)
\end{aligned}
$$

where

$$
\begin{aligned}
\mathcal{Q}_{p}\left(\kappa_{1}, \kappa_{2} ; a_{1}, a_{2}\right)= & a_{1}^{3}\left[\kappa_{1}\left(p^{2}-2 p+2\right)+2\left(\kappa_{2}+2\right)(p-1)\right]+a_{2}^{3}\left(\kappa_{1}-\kappa_{2}\right) \\
& +a_{1}^{2} a_{2}(p-2)\left[\kappa_{1}(p-2)+2\left(\kappa_{2}+2\right)\right] \\
& +a_{1} a_{2}^{2}\left[\kappa_{1}(3-2 p)+\kappa_{2}(p-3)-4\right] \\
\mathcal{R}_{p}\left(\kappa_{1}, \kappa_{2} ; a_{1}, a_{2}\right)= & a_{1}^{3}\left[p^{2}+2(p-1)\left(\kappa_{1}+\kappa_{2}\right)\right]+a_{1}^{2} a_{2}(p-2)\left[2\left(\kappa_{1}+\kappa_{2}\right)+p\right] \\
& -a_{1} a_{2}^{2}\left[2\left(\kappa_{1}+\kappa_{2}\right)+p\right] .
\end{aligned}
$$


Using this formula we recover $I^{\mathrm{IR}}\left[\right.$ Bolt $\left._{ \pm}\right]$in (3.26) with $\kappa_{1}=\kappa_{2}=\mp 1$ and $a_{1}=0$. Similarly, we recover $I^{\mathrm{IR}}\left[\right.$ Bolt $\left._{ \pm}\right]$in (3.37) when substituting $\kappa_{1}=-\kappa_{2}=\mp 1$ and

$$
a_{1}=-2\left(2 s+\sqrt{4 s^{2}-1}\right), \quad a_{2}=-2\left(\frac{p}{4 s}-2 s-\sqrt{4 s^{2}-1}\right) .
$$

The conformal boundary of $Y_{4}$ is a squashed Lens space $L(p, 1) \cong S^{3} / \mathbb{Z}_{p}$, with fibration determined by the choice of Killing vector. Thus, the concrete expression for (2.35) will depend on $a_{1}, a_{2}$. However, we can also consider more general vector spaces $L(p, q)$, which are toric 3-manifolds. Thus, the toric geometry techniques used earlier for $\mathcal{O}(-p) \rightarrow S^{2}$ can be applied to this case as well, assuming that the torus action extends to the bulk. The case considered in [7] is that of $L(3,2)$, for which we expect three nuts with equal chiralities $\kappa$ :

$$
\begin{aligned}
I_{L(3,2)}^{\mathrm{IR}} & =\sum_{i=1}^{3}-\kappa_{i}\left(\frac{\pi}{2 G_{4}}-\kappa_{i} \frac{\mathrm{i} \vartheta}{2}\right) \frac{\left(b_{1}^{(i)}-\kappa_{i} b_{2}^{(i)}\right)^{2}}{4 b_{1}^{(i)} b_{2}^{(i)}} \\
& =\left(\kappa \frac{\pi}{2 G_{4}}-\frac{\mathrm{i} \vartheta}{2}\right) \frac{3\left(2 a_{1}\left(a_{1}-a_{2}\right)(1+\kappa)-a_{2}^{2}(3+4 \kappa)\right)}{4\left(a_{1}-2 a_{2}\right)\left(a_{1}+a_{2}\right)}
\end{aligned}
$$

One of the interesting features of this is example is that $L(3,2)$ is homeomorphic to $L(3,1)$. The action of the filling of the latter can be computed using (3.38), obtaining a different result and showing the effects of different supersymmetric structures even for topologically equivalent backgrounds

$$
I_{L(3,1)}^{\mathrm{IR}}=\left(\kappa \frac{\pi}{2 G_{4}}-\frac{\mathrm{i} \vartheta}{2}\right) \frac{3\left(a_{1}^{2}(9+8 \kappa)+a_{1} a_{2}(3+4 \kappa)-a_{2}^{2}(3+4 \kappa)\right)}{4\left(2 a_{1}-a_{2}\right)\left(a_{1}+a_{2}\right)} .
$$

Given any four-manifold with torus action, we can also investigate the behaviour of the on-shell action and the $\vartheta$ term under blow-up, corresponding to substituting a point in $\mathbb{R}^{4}$ with $\mathcal{O}(-1) \rightarrow S^{2}$. Using the toric geometry description in [7], it is easy to see that if the original nut had chirality $\kappa$ and the solution had on-shell action $I$, then the action of the solution with blown-up topology is

$$
I_{\text {blow up }}=I+\frac{2+3 \kappa}{4}\left(\frac{\pi}{2 G_{4}}-\kappa \frac{\mathrm{i} \vartheta}{2}\right) .
$$

Indeed, the $\frac{1}{4}$-BPS solution Bolt + with topology $\mathcal{O}(-1) \rightarrow S^{2}$ can be viewed as the blow up of the $\frac{1}{4}$-BPS Taub-NUT-AdS solution (the former has a bolt - and the latter a nut_), and the results for the on-shell action match.

\section{Origin of the $\vartheta$ term}

Four-dimensional minimal supergravity arises from the consistent truncation of ten- and eleven-dimensional supergravities on numerous six- and seven-dimensional internal spaces (potentially with fluxes). In the context of top-down constructions of the AdS/CFT correspondence, different choices of internal manifolds and fluxes correspond to different field theories, so the four-dimensional minimal supergravity describes the "universal" part of the dual three-dimensional $\mathcal{N}=2$ SCFT corresponding to each internal manifold. 
Here we focus on three-dimensional SCFTs constructed from stacks of $M$-branes in $M$-theory. The corresponding dual geometries are eleven-dimensional space-times with an $\mathrm{AdS}_{4}$ factors, and have been analysed in some generality (see e.g. [58]). Each of these geometries should correspond to the uplift of the vacuum solution of an effective fourdimensional gauged supergravity obtained by consistently truncate the eleven-dimensional supergravity on the seven-dimensional internal space [14].

We only consider the truncation of the bosonic sector of eleven-dimensional supergravity, which contains the eleven-dimensional metric $g\left(Y_{11}\right)$ and a three-form gauge field $C$, with curvature $G=\mathrm{d} C$, interacting via

$$
S_{11}=\frac{1}{2 \kappa_{11}^{2}} \int\left(R\left(Y_{11}\right) \operatorname{vol}\left(Y_{11}\right)-\frac{1}{2} G \wedge *_{11} G-\alpha \frac{1}{6} C \wedge G \wedge G\right)
$$

and $2 \kappa_{11}^{2}=(2 \pi)^{8} \ell_{P}^{9}$. For the purposes of this section we move back to Lorentzian signature and reinstate the gauge couplings. The sign of $\alpha= \pm 1$ is determined by the supersymmetry conventions.

As mentioned above, it is possible to obtain four-dimensional minimal gauged supergravity also via consistent truncations of ten-dimensional supergravity. An intriguing possibility is the compactification from massive IIA, which itself breaks parity. In this case, the internal space is a smooth geometry over a space that is topologically $S^{6}$, constructed as a $S^{2}$ bundle over $\mathbb{C P}^{2}[59,60]$. The dual field theories are SCFTs obtained from $D 2$ branes. Note that the smoothness condition can be relaxed, obtaining internal spaces that consist of singular geometries constructed by replacing the $\mathbb{C P}^{2}$ base of the bundle with another Kähler-Einstein space [61].

\subsection{M2-branes}

For the case of field theories obtained from arrangements of $M 2$ branes, there are numerous consistent truncations known. Equivalently, it is known that for any supersymmetric solution to the equations of motion of minimal gauged supergravity (2.2) there are numerous ways of uplifting on a seven-dimensional manifold to obtain an eleven-dimensional configuration for which the integral of $*_{11} G$ through a seven-cycle is non-zero, see e.g. [14, 15, 62]. Here we consider one such ansatz. Inspired by the Freund-Rubin $\mathrm{AdS}_{4} \times S^{7}$ solution, we take the ansatz $Y_{4} \times S E_{7}$, where $Y_{4}$ is the four-dimensional spacetime and $S E_{7}$ is a sevendimensional Sasaki-Einstein manifold [14]. ${ }^{6}$ Locally, we may write the eleven-dimensional metric as

$$
\begin{aligned}
g\left(Y_{11}\right) & =\frac{L^{2}}{\mathfrak{g}^{2}}\left[\frac{\mathfrak{g}^{2}}{4} g\left(Y_{4}\right)+\left(\mathrm{d} \psi+\sigma+\frac{\mathfrak{g}}{2} A\right)^{2}+g\left(N_{6}\right)\right], \\
G & =\frac{L^{3}}{\mathfrak{g}^{3}}\left[\frac{3 \mathfrak{g}^{4}}{8} \operatorname{vol}\left(Y_{4}\right)-\frac{\mathfrak{g}}{2} *_{4} F \wedge J\right]
\end{aligned}
$$

where the second factor is a fibering over the local form of the metric on $S E_{7}$ : $\partial_{\psi}$ is the Reeb vector, $J$ is the Kähler form on the local Kähler-Einstein space $N_{6}$ over which $S E_{7}$

\footnotetext{
${ }^{6}$ There are restrictions on the global structure of the $S E_{7}$, depending on the topology and spin ${ }^{c}$ structure on $Y_{4}[2,31]$. The supersymmetry conventions here are such that $\alpha=+1$.
} 
is constructed. $A$ is a $\mathrm{U}(1)$ gauge connection with curvature $F=\mathrm{d} A$. The metric on $S E_{7}$ is normalized so that $\operatorname{Ric}\left(g\left(S E_{7}\right)\right)=6 g\left(S E_{7}\right)$.

This ansatz gives a consistent truncation of eleven-dimensional supergravity down to minimal four-dimensional supergravity, in the sense that upon substitution in the equations of motion obtained from the action (4.1), we obtain the equations of motion (2.2). Once this has been checked, we may insert the ansatz back in the action $S_{11}$ : could there be a $\vartheta$ term from the eleven-dimensional topological term? We have

$$
G \wedge G=-\frac{L^{6}}{4 \mathfrak{g}^{4}} J \wedge J \wedge F \wedge F
$$

so to find a $\vartheta$ term in four dimensions we would need a term $\Psi_{3}$ in $C$ entirely along $S E_{7}$, which is only compatible with the ansatz (4.2) if such term is closed. In order to have a non-trivial integral on $S E_{7}$ when reducing, we need $\left.\partial_{\psi}\right\lrcorner \Psi_{3} \neq 0$. However, it is easy to see that this requirement cannot be combined with closedness, in the sense that together they imply that $\Psi_{3} \wedge G \wedge G=0$. In fact, this argument can be extended to different Freund-Rubin-type reductions: it is not possible to include a non-trivial pullback $\left.G\right|_{S E_{7}}$, and thus have a non-trivial $\left.C\right|_{S E_{7}}$ [63]. Therefore, we conclude that generically there is no $\vartheta$ term in the supergravity action obtained by reduction of eleven-dimensional supergravity on $S E_{7}$, which is holographically dual to three-dimensional SCFTs with $\mathcal{N}=2$ obtained from $M 2$-branes.

In order to compare the field theory and gravity observables, the uplift (4.2) gives the relation between the four-dimensional Newton constant and the quantization of the fluxes for $G$. Consistently with the interpretation as gravity dual to $M 2$ branes, $G$ has an electric flux proportional to $\operatorname{vol}\left(Y_{4}\right)$, and

$$
N_{M 2}=-\frac{1}{\left(2 \pi \ell_{P}\right)^{6}} \int_{S E_{7}} *{ }_{11} G=\frac{6 L^{6} \operatorname{Vol}\left(S E_{7}\right)}{\mathfrak{g}^{6}\left(2 \pi \ell_{P}\right)^{6}}
$$

Combining with the reduction of the Ricci scalar, we find

$$
\frac{1}{16 \pi G_{4}}=\frac{\pi \mathfrak{g}^{2}}{12 \sqrt{6 \mathrm{Vol}\left(S E_{7}\right)}} N_{M 2}^{3 / 2}
$$

This indeed reproduces the large $N$ behaviour $N^{3 / 2}$ typical of $M 2$ branes.

\section{$4.2 \quad M 5$-branes}

The other maximally symmetric Freund-Rubin solution $\operatorname{AdS}_{7} \times S^{4}$ is dual to the worldvolume theory of a stack of $M 5$-branes. The corresponding consistent truncation was found in $[64,65] .{ }^{7}$ Using the notations of [67], we write the eleven-dimensional metric as

$$
g\left(Y_{11}\right)=\frac{\Delta^{1 / 3}}{\mathfrak{g}^{2}}\left[\mathfrak{g}^{2} g\left(Y_{7}\right)+\Delta^{-1} T_{i j}^{-1} D \mu^{i} D \mu^{j}\right]
$$

\footnotetext{
${ }^{7}$ Given the importance of the gauge fields in the following, we should point out that the consistent truncation from eleven to seven dimensions has only been worked out in full with the gauge fields set to zero. More precisely, the reduction of the eleven-dimensional Einstein equations of motion has been done with vanishing gauge fields. Nonetheless, there is substantial supporting evidence in favour of the consistency, including the full reduction of the supersymmetry variations [65], the reduction of less supersymmetric supergravities [66], and various limits of the ansatz, such as [67]. In this case, we should pick $\alpha=-1$ in (4.1) to be consistent with the supersymmetry conventions.
} 
and the four-form as

$$
\begin{aligned}
G= & \frac{\Delta^{-2}}{\mathfrak{g}^{3} 4 !} \epsilon_{i_{1} \ldots i_{5}}\left(-U \mu^{i_{1}} D \mu^{i_{2}} \wedge D \mu^{i_{3}} \wedge D \mu^{i_{4}} \wedge D \mu^{i_{5}}\right. \\
& +4 T^{i_{1} m} D T^{i_{2} n} \wedge \mu^{m} \mu^{n} D \mu^{i_{3}} \wedge D \mu^{i_{4}} \wedge D \mu^{i_{5}} \\
& \left.+6 \mathfrak{g} \Delta \mathcal{F}^{i_{1} i_{2}} \wedge D \mu^{i_{3}} \wedge D \mu^{i_{4}} T^{i_{5} j} \mu^{j}\right) \\
& -T_{i j} *_{7} S_{(3)}^{i} \mu^{j}+\frac{1}{\mathfrak{g}} S_{(3)}^{i} \wedge D \mu^{i}
\end{aligned}
$$

In these expressions, $\mathfrak{g}$ is the gauge coupling, $i, j=1, \ldots 5$, and we have introduced the following objects: coordinates $\mu^{i}$ on $S^{4}$ satisfying $\sum \mu^{i} \mu^{i}=1$; three-forms $S_{(3)}^{i}$; $\mathrm{SO}(5)$ gauge fields $\mathcal{A}_{(1)}^{i j}$ with curvature $\mathcal{F}_{(2)}^{i j}$; and fourteen scalar fields grouped in the symmetric unimodular matrix $T^{i j}$. Moreover, we have defined

$$
\begin{aligned}
\Delta & \equiv T_{i j} \mu^{i} \mu^{j}, & U & \equiv 2 T_{i j} T_{j k} \mu^{i} \mu^{k}-\Delta T_{i i}, \\
D \mu^{i} & =\mathrm{d} \mu^{i}+\mathfrak{g} \mathcal{A}^{i j} \mu^{j}, & D T_{i j} & =\mathrm{d} T_{i j}+\mathfrak{g} \mathcal{A}^{i k} T_{k j}+\mathfrak{g} \mathcal{A}^{j k} T_{k i}, \\
D S_{(3)}^{i} & =\mathrm{d} S_{(3)}^{i}+\mathfrak{g} \mathcal{A}^{i j} \wedge S_{(3)}^{j}, & \mathcal{F}^{i j} & =\mathrm{d} \mathcal{A}^{i j}+\mathfrak{g} \mathcal{A}^{i k} \wedge \mathcal{A}^{k j} .
\end{aligned}
$$

Inserting this ansatz into the equations of motion and action of eleven-dimensional supergravity takes us to the $\mathrm{SO}(5)$ gauged maximal supergravity in seven dimensions [68]

$$
\begin{aligned}
S_{7}= & \frac{1}{2 \kappa_{7}^{2}} \int\left[(R-V) \operatorname{vol}\left(Y_{7}\right)-\frac{1}{4} T_{i j}^{-1} D T_{j k} \wedge *_{7} T_{k l}^{-1} D T_{l i}-\frac{1}{4} T_{i k}^{-1} T_{j l}^{-1} \mathcal{F}^{i j} \wedge *_{7} \mathcal{F}^{k l}\right. \\
& -\frac{1}{2} T_{i j} S_{(3)}^{i} \wedge *_{7} S_{(3)}^{j}+\frac{1}{2 \mathfrak{g}} S_{(3)}^{i} \wedge D S_{(3)}^{i}-\frac{1}{8 \mathfrak{g}} \epsilon_{i j_{1} \ldots j_{4}} S_{(3)}^{i} \wedge \mathcal{F}^{j_{1} j_{2}} \wedge \mathcal{F}^{j_{3} j_{4}} \\
& \left.+\frac{1}{16 \mathfrak{g}} Q_{3}[\mathcal{A}, \mathcal{F}] \wedge P_{4}[\mathcal{F}]-\frac{1}{8 \mathfrak{g}} Q_{7}[\mathcal{A}, \mathcal{F}]\right]
\end{aligned}
$$

where the scalar potential is

$$
V=\frac{1}{2} \mathfrak{g}^{2}\left(2 T_{i j} T_{i j}-\left(T_{i i}\right)^{2}\right)
$$

and the seven-dimensional Chern-Simons forms are given by

$$
\begin{aligned}
Q_{3}[A, F] \wedge P_{4}[F]= & \operatorname{tr}\left(A \wedge F-\frac{1}{3} \mathfrak{g} A \wedge A \wedge A\right) \wedge \operatorname{tr}(F \wedge F) \\
Q_{7}[A, F]= & \operatorname{tr}\left(A \wedge F \wedge F-\frac{2}{5} \mathfrak{g} A \wedge A \wedge A \wedge F \wedge F-\frac{1}{5} \mathfrak{g} A \wedge F \wedge A \wedge A \wedge F\right. \\
& +\frac{1}{5} \mathfrak{g}^{2} A \wedge A \wedge A \wedge A \wedge A \wedge F \\
& \left.-\frac{1}{35} \mathfrak{g}^{3} A \wedge A \wedge A \wedge A \wedge A \wedge A \wedge A\right)
\end{aligned}
$$

Solutions to this supergravity uplift to eleven-dimensional solutions, and describe holographic duals to the six-dimensional $(2,0)$ SCFT on spaces diffeomorphic to their conformal boundary. The relation between the eleven-dimensional and seven-dimensional Newton 
constants is found by looking at the reduction of the action:

$$
\frac{1}{2 \kappa_{7}^{2}}=\frac{\operatorname{Vol}\left(S^{4}\right)}{(2 \pi)^{8} \ell_{P}^{9} \mathfrak{g}^{4}}
$$

Compared with the previous ansatz, we immediately see that $G$ satisfies a different flux quantisation condition, corresponding to the presence of $M 5$ branes $^{8}$

$$
N_{M 5}=\frac{1}{\left(2 \pi \ell_{P}\right)^{3}} \int_{S^{4}} G=\frac{1}{\left(2 \pi \ell_{P}\right)^{3}} \int_{S^{4}}\left(-\frac{\Delta^{-2} U}{\mathfrak{g}^{3}} \operatorname{vol}\left(S^{4}\right)\right) .
$$

Combining the two, we find

$$
\frac{1}{2 \kappa_{7}^{2}}=2 \pi \mathfrak{g}^{5} \frac{\operatorname{Vol}\left(S^{4}\right)}{3\left(-\int_{S^{4}} \Delta^{-2} U \operatorname{vol}\left(S^{4}\right)\right)^{3}} N_{M 5}^{3}
$$

For instance, the vacuum solution has metric $g\left(Y_{7}\right)=4 g\left(A d S_{7}\right) / \mathfrak{g}^{2}$, where $g\left(A d S_{7}\right)$ is normalised to have sectional curvature equal to -1 , and non-vanishing $T_{i j}=\delta_{i j}$. It uplifts to eleven dimensions on $S^{4}$ as the Freund-Rubin solution, that is, without fibering and warping, and

$$
\frac{1}{2 \kappa_{7}^{2}}=\frac{\mathfrak{g}^{5}}{96 \pi^{3}} N_{M 5}^{3}
$$

Our interest, though, is in supersymmetric solutions containing a three-dimensional hyperbolic factor, such as that found in $[70]^{9}$

$$
\begin{aligned}
g\left(Y_{7}\right) & =\frac{2^{\frac{4}{5}}}{\mathfrak{g}^{2}}\left[g\left(\mathrm{AdS}_{4}\right)+g\left(\Sigma_{3}\right)\right], \quad T=\operatorname{diag}\left(2^{-\frac{2}{5}}, 2^{-\frac{2}{5}}, 2^{-\frac{2}{5}}, 2^{\frac{3}{5}}, 2^{\frac{3}{5}}\right) \\
\mathcal{A}^{a b} & =\frac{1}{\mathfrak{g}} \omega^{a b},
\end{aligned}
$$

where $\Sigma_{3}$ is a closed hyperbolic 3-manifold, and both $g\left(\mathrm{AdS}_{4}\right)$ and $g\left(\Sigma_{3}\right)$ are normalized to have constant sectional curvature equal to -1 . We have broken $\mathrm{SO}(5)$ to $\mathrm{SO}(3) \times$ $\mathrm{SO}(2)$, splitting the indices $i, j$ into $a, b=1,2,3$ and $\alpha, \beta=1,2$, and set the $\mathrm{SO}(3)$ gauge fields proportional to the spin connection $\omega$ of $\Sigma_{3}$. This solution describes $M 5$ branes wrapped around $\Sigma_{3}$, which is showed by the presence of a gauge field fixed by the geometry, corresponding in the field theory setup to a non-trivial background gauge field realizing the topological twist [71].

As we always expect, the presence of this solution signals the existence of a consistent truncation to an effective four-dimensional gauged supergravity with anti-de Sitter vacuum. In fact, there is a consistent truncation to four-dimensional $\mathcal{N}=2$ gauged supergravity

${ }^{8}$ Generically, it is not $G$ that has quantised flux, but rather [69]

$$
\left[\frac{1}{\left(2 \pi \ell_{P}\right)^{3}} G\right]-\frac{1}{4} p_{1}\left(Y_{11}\right) \in H^{4}\left(Y_{11}, \mathbb{Z}\right) .
$$

However, the Pontryagin class of any spin manifold of dimension equal or lower than seven is a multiple of 4 , so the quantization condition is not corrected for our purposes.

${ }^{9}$ There is also a non-supersymmetric vacuum anti-de Sitter solution, which is not relevant for us. 
with one vector multiplet and two hypermultiplets [72], but here we will only be concerned with a truncation to minimal gauged supergravity. It is obtained by the following ansatz

$$
\begin{aligned}
g\left(Y_{7}\right) & =\frac{2^{\frac{4}{5}}}{\mathfrak{g}^{2}}\left[\mathfrak{g}^{2} g\left(Y_{4}\right)+g\left(\Sigma_{3}\right)\right], & T & =\operatorname{diag}\left(2^{-\frac{2}{5}}, 2^{-\frac{2}{5}}, 2^{-\frac{2}{5}}, 2^{\frac{3}{5}}, 2^{\frac{3}{5}}\right), \\
\mathcal{A}^{a b} & =\frac{1}{\mathfrak{g}} \omega^{a b}, \quad \mathcal{A}^{\alpha \beta}=2 \epsilon^{\alpha \beta} A, & S_{(3)}^{a} & =-\frac{2}{\mathfrak{g}} *_{4} F \wedge \mathrm{e}^{a},
\end{aligned}
$$

where $A$ is a $\mathrm{U}(1)$ gauge field on $Y_{4}$ with curvature $F=\mathrm{d} A, \mathrm{e}^{a}$ and $\omega^{a b}$ are the dreibein and spin connection of the hyperbolic metric on $\Sigma_{3}$. Inserting this ansatz into the equations of motion derived from (4.9), leads to the equations of motion of four-dimensional minimal gauged supergravity (2.2). Notice for instance that the vacuum solution $g\left(A d S_{4}\right) / \mathfrak{g}^{2}$ with vanishing gauge field uplifts to the Pernici-Sezgin solution (4.17).

Having showed consistency, we can then substitute the ansatz back inside the action (4.9). Whilst the first two lines give an expression proportional to $\operatorname{Vol}\left(\Sigma_{3}\right)$, the topological term in the last line of (4.9) results in a purely topological term in four dimensions as well. To be more precise, we write a bulk manifold $X_{8} \cong X_{4} \times Y_{4}$ with $\partial X_{4} \cong \Sigma_{3}$, so clearly $\partial X_{8} \cong Y_{7}$. Then the seven-dimensional topological term may be written as

$$
\begin{aligned}
\frac{1}{2 \kappa_{7}^{2}} \frac{1}{16 \mathfrak{g}} \int_{X_{8}}\left(P_{4}[\mathcal{F}]^{2}-2 P_{8}[\mathcal{F}]\right) & =-\frac{1}{2 \kappa_{7}^{2} \mathfrak{g}^{3}} \int_{X_{4}} P_{4}[\bar{\rho}] \int_{Y_{4}} F \wedge F \\
& =-\frac{8 \pi c s\left(\Sigma_{3}\right)}{2 \kappa_{7}^{2} \mathfrak{g}^{3}} \int_{Y_{4}} F \wedge F
\end{aligned}
$$

Here, $\bar{\rho}$ is the extension in the bulk $X_{4}$ of the curvature two-form of $\omega$, and $\operatorname{cs}\left(\Sigma_{3}\right)$ is the three-dimensional Chern-Simons invariant of the three-manifold $\Sigma_{3}$

$$
\operatorname{cs}\left(\Sigma_{3}\right)=\frac{1}{8 \pi} \int_{\Sigma_{3}} \operatorname{tr}_{3}\left(\omega \wedge \mathrm{d} \omega+\frac{2}{3} \omega \wedge \omega \wedge \omega\right),
$$

where the trace is in the defining representation of $\mathfrak{s o}(3)$. It is defined modulo $2 \pi$ having chosen a spin structure on $\Sigma_{3}$ (see appendix A).

The main difference with the $M 2$-brane ansatz (4.2) is that now we find an additional $\vartheta$ term due to the reduction of the seven-dimensional topological terms

$$
S_{7}=\frac{4 \operatorname{Vol}\left(\Sigma_{3}\right)}{2 \kappa^{7} \mathfrak{g}^{3}} \int_{Y_{4}}\left(R\left(g\left(Y_{4}\right)\right)+6 \mathfrak{g}^{2}-F^{2}\right) \operatorname{vol}\left(Y_{4}\right)-\frac{8 \pi c s\left(\Sigma_{3}\right)}{2 \kappa_{7}^{2} \mathfrak{g}^{3}} \int_{Y_{4}} F \wedge F .
$$

In order to find the relation between the four-dimensional gravitational constants and the M-theory quantities, we need an expression for $\Delta$ and $U$ to substitute in (4.15). To find that, we plug the ansatz (4.18) in the uplift (4.6). To be more concrete, following [17, 21], we introduce the following coordinates $\mu^{i}$ on $S^{4}$ :

$$
\begin{aligned}
\mu^{a} & =\cos (\nu) \widetilde{\mu}^{a}, & & a=1,2,3, \\
\mu^{\alpha} & =\sin (\nu) \widetilde{\mu}^{\alpha}, & \alpha & =4,5,
\end{aligned}
$$

with $\sum_{i=1}^{5}\left(\mu^{i}\right)^{2}=\sum_{a=1}^{3}\left(\widetilde{\mu}^{a}\right)^{2}=\sum_{\alpha=4}^{5}\left(\widetilde{\mu}^{\alpha}\right)^{2}=1$. Furthermore, we use the explicit parametrization

$$
\begin{aligned}
& \tilde{\mu}^{1}=\cos \xi_{1}, \quad \tilde{\mu}^{2}=\sin \xi_{1} \cos \xi_{2}, \quad \tilde{\mu}^{3}=\sin \xi_{1} \sin \xi_{2}, \\
& \tilde{\mu}^{4}=\cos \psi, \quad \tilde{\mu}^{5}=\sin \psi .
\end{aligned}
$$


with coordinate ranges $0 \leq \nu \leq \pi / 2,0 \leq \psi<\Delta \psi, 0 \leq \xi_{1} \leq \pi$, and $0 \leq \xi_{2}<2 \pi$. Using these coordinates, we have

$$
\Delta=2^{-2 / 5}\left(1+\sin ^{2} \nu\right), \quad U=-2^{-4 / 5}\left(5+\sin ^{2} \nu\right) .
$$

The eleven-dimensional ansatz for the metric is written

$$
\begin{aligned}
g\left(Y_{11}\right)= & \frac{2^{\frac{2}{3}}\left(1+\sin ^{2} \nu\right)^{\frac{1}{3}}}{\mathfrak{g}^{2}}\left[\mathfrak{g}^{2} g\left(Y_{4}\right)+g\left(\Sigma_{3}\right)+\frac{1}{2}\left(\mathrm{~d} \nu^{2}+\frac{\sin ^{2} \nu}{1+\sin ^{2} \nu}(\mathrm{d} \psi-\mathfrak{g} A)^{2}\right)\right. \\
& \left.+\frac{\cos ^{2} \nu}{1+\sin ^{2} \nu} \sum_{a=1}^{3}\left(\mathrm{~d} \widetilde{\mu}^{a}+\omega^{a b} \widetilde{\mu}^{b}\right)^{2}\right],
\end{aligned}
$$

and the four-form gauge field is

$$
\begin{aligned}
G= & \frac{\left(5+\sin ^{2} \nu\right)}{\mathfrak{g}^{3}\left(1+\sin ^{2} \nu\right)^{2}} \epsilon_{a b c} \epsilon_{\alpha \beta} D \mu^{b} \wedge D \mu^{c} \wedge\left(\frac{1}{4} \mu^{a} D \mu^{\alpha} \wedge D \mu^{\beta}+\frac{1}{6} \mu^{\alpha} D \mu^{\beta} \wedge D \mu^{a}\right) \\
& +\frac{\epsilon_{a b c} \epsilon_{\alpha \beta}}{\mathfrak{g}^{3}\left(1+\sin ^{2} \nu\right)} \rho^{a b} \wedge\left(D \mu^{c} \wedge D \mu^{\alpha} \mu^{\beta}+\frac{1}{4} D \mu^{\alpha} \wedge D \mu^{\beta} \mu^{c}\right) \\
& +\frac{\epsilon_{a b c}}{\mathfrak{g}^{2}\left(1+\sin ^{2} \nu\right)} F \wedge D \mu^{a} \wedge D \mu^{b} \mu^{c}-\frac{2}{\mathfrak{g}^{2}} *_{4} F \wedge \mathrm{e}^{a} \wedge D \mu^{a}-\frac{2}{\mathfrak{g}^{2}} F \wedge *_{3} \mathrm{e}^{a} \mu^{a},
\end{aligned}
$$

where $\rho^{a b}=-\mathrm{e}^{a} \wedge \mathrm{e}^{b}$ is the curvature two-form of the spin connection for $\Sigma_{3}$.

Substituting these values in (4.15) and in turn in (4.21) leads to

$$
S=\frac{1}{16 \pi G_{4}} \int\left(R_{g}+6 \mathfrak{g}^{2}-F^{2}\right) \operatorname{vol}_{g}-\frac{\vartheta \mathfrak{g}^{2}}{8 \pi^{2}} \int F \wedge F
$$

with

$$
\frac{1}{G_{4}}=\frac{8 \operatorname{Vol}\left(\Sigma_{3}\right) \mathfrak{g}^{2}}{3 \Delta \psi^{2}} N_{M 5}^{3}, \quad \vartheta=\frac{8 \pi^{2} \operatorname{cs}\left(\Sigma_{3}\right)}{3 \Delta \psi^{2}} N_{M 5}^{3} .
$$

This reproduces the large $N$ behaviour typical of $M 5$-branes.

\subsection{Uplift: topological considerations}

The eleven-dimensional solution is constructed as a fibration of $S^{4}$ over $Y_{4}$ and $\Sigma_{3}$. The choice of $\Sigma_{3}$ corresponds to the choice of field theory. A necessary requirement is that the full solution must be spin.

If we choose the eleven-dimensional solution to be globally a fibration with $\Sigma_{3}$ and $S^{4}$ - which are spin - then $Y_{4}$ is also required to be spin and so $A$ must be a connection on a $\mathrm{U}(1)$ bundle. This is fine for many solutions, including $\mathrm{AdS}_{4}$ (even with the non-trivial instanton of $[50,51])$; the self-dual Taub-NUT-AdS solutions of [31], which have topology $\mathbb{R}^{4}$; the SUSY dyonic black holes with topology $D^{2} \times \Sigma_{g}$.

However, generically there are also four-dimensional solutions $Y_{4}$ that are only $\operatorname{spin}^{c}$. For instance, this is the case for the non-self-dual bolt solutions of [31]. They have topology $\mathcal{M}_{p} \equiv \mathcal{O}(-p) \rightarrow \Sigma_{g}$ and the U(1) connection has flux through the $\Sigma_{g}$ cycle. For the $\frac{1}{4}$-BPS, we have

$$
\mathfrak{g} \int_{\Sigma_{g}} \frac{F}{2 \pi}= \pm \frac{p}{2}-(1-g) .
$$

Since this is a half-integer for odd $p, A$ is a $\operatorname{spin}^{c}$ field in that case. 
Looking at the form of the metric in (4.25), we see that the $S^{4}$ is written as a $S^{1} \times S^{2}$ fibration over an interval: at $\nu=0$ the circle collapses and the $S^{2}$ collapses at $\nu=\pi / 2$. In order to guarantee the regularity of the metric at $\nu=0$ it is important that the global angular form of the circle bundle has the right periodicity. The canonical periodicity of $\psi$ in order to cover the full $S^{4}$ is $\Delta \psi=2 \pi$. To have a well-defined circle bundle its first Chern number should be an integer, which would also require $A$ to be a well-defined U(1) connection, which doesn't happen for generic $Y_{4}=\mathcal{M}_{p}$. To remedy this and guarantee that we still have a well-defined circle bundle, we should change the periodicity of $\psi$ : by setting $\Delta \psi=2 \pi / k$, the first Chern number of the bundle over $\Sigma_{g}$ is

$$
\begin{aligned}
k \mathfrak{g} \int_{\Sigma_{g}} \frac{F}{2 \pi} \in \mathbb{Z} & \Leftrightarrow \quad k\left( \pm \frac{p}{2}-(1-g)\right) \in \mathbb{Z} \\
& \Leftrightarrow \quad \pm k p-2 k(1-g)=0 \quad \bmod 2 .
\end{aligned}
$$

This requires $k p \in 2 \mathbb{Z}$ : if $p$ is odd, we need to take $k=2$, for instance. In fact, this is more general than this specific solution: if $A$ is a $\operatorname{spin}^{c}$ connection, we require $\psi$ to have $\pi$ periodicity, resulting in uplifting $Y_{4}$ on $\Sigma_{3} \times S^{4} / \mathbb{Z}_{2}$.

Having considered the global regularity of the metric, we should then ask whether the eleven-dimensional space $Y_{11}$ is spin. As mentioned, $Y_{11}$ is a sphere bundle of a $\mathbb{R}^{5}$ bundle over $Y_{4} \times \Sigma_{3}$, with total space $Z_{12}$. In fact, the way $S^{4}$ is written in (4.25) shows that it is easier to write $\mathbb{R}^{5} \cong \mathbb{R}^{2} \times \mathbb{R}^{3}$, where $\mathbb{R}^{2}$ is fibered over $Y_{4}$ and $\mathbb{R}^{3}$ is fibered over $\Sigma_{3}$. Since $Y_{11} \cong \partial Z_{12}$, we may focus on $Z_{12}$. More is true: any vector bundle deformation retracts to its zero section, which is isomorphic to the base, so we can just study $Y_{4} \times \Sigma_{3}$. This is because a deformation retraction is an homotopy equivalence, so $H^{\bullet}\left(Z_{12}\right) \cong H^{\bullet}\left(Y_{4} \times \Sigma_{3}\right)$.

The bundle we should focus on is then $\mathcal{L} \oplus T \Sigma_{3}$, where $\mathcal{L}$ is the $\mathbb{R}^{2}$ bundle over $Y_{4}$. It is tempting to conclude that $Z_{12}$ is spin iff $\mathcal{L}$ has vanishing Stiefel-Whitney class, but there is a subtlety that we have so far glossed over. As we have found earlier, in order to guarantee regularity of the metric it is necessary to uplift $\operatorname{spin}^{c}$ manifolds on $S^{4} / \mathbb{Z}_{2}$, which means that in those cases $\mathcal{L}$ is a bundle with fiber $\mathbb{R}^{2} / \mathbb{Z}_{2}$. Studying its cohomology is subtler, and we shall not attempt it here. We should remark, though, that asking for spin $Z_{12}$ is only necessary in order to guarantee that $Y_{11}$ is spin, so it could be possible to find the uplift conditions in another way. In the following we shall restrict our considerations to spin $Y_{4}$, which uplift on $S^{4}$.

\subsection{Subleading corrections}

Starting from eleven-dimensional supergravity, we can also begin studying the subleading corrections to the large $N$ result. As before, we first reduce to seven-dimensional supergravity, and then to four-dimensional minimal supergravity using the Pernici-Sezgin ansatz. However, in order to simplify our computations we shall focus only on the $\mathrm{SO}(5)$ gauge fields, partly following [73]. As mentioned in the previous section, we view spacetime $Y_{11}$ as a sphere bundle over $Y_{7}$, and the resulting seven-dimensional gauge group arising from the structure group of this bundle. The ansatz for the four-form (4.7), which has non-trivial flux through the four-sphere, is an extension of the global angular four-form, which is the 
unique closed and gauge-invariant extension of the sphere volume form (see appendix B). In fact, we may single out the part of the ansatz with flux through $S^{4}$ by writing ${ }^{10}$

$$
G=\frac{8 \pi^{2}}{\mathfrak{g}^{3}} \mathrm{E}_{4}+\ldots
$$

A more precise definition of the topological term in the action of eleven-dimensional supergravity (4.1) can be given in terms of the integral of a closed form on a twelve-dimensional manifold $X_{12}$ for which $\partial X_{12}=Y_{11}$, which we view as a $S^{4}$ fibration over $X_{8}$ (with $\partial X_{8} \cong Y_{7}$ ). Then, we can use the Bott-Cattaneo formula (B.6)

$$
\begin{aligned}
S_{11} & \supset-\alpha \frac{1}{2 \kappa_{11}^{2}} \frac{1}{6} \int_{X_{12}} G \wedge G \wedge G=-\alpha \frac{1}{2 \kappa_{11}^{2}} \frac{256 \pi^{6}}{3 \mathfrak{g}^{9}} \int_{X_{12}} \mathrm{E}_{4} \wedge \mathrm{E}_{4} \wedge \mathrm{E}_{4} \\
& =-\alpha \frac{1}{2 \kappa_{11}^{2}} \frac{64 \pi^{6}}{3 \mathfrak{g}^{5}} \int_{X_{8}} p_{2}(\bar{E}) \\
& =-\alpha \frac{1}{2 \kappa_{7}^{2}} \frac{1}{16 \mathfrak{g}} \int_{Y_{7}}\left(Q_{3}[\mathcal{A}, \mathcal{F}] \wedge P_{4}[\mathcal{F}]-2 Q_{7}[\mathcal{A}, \mathcal{F}]\right) .
\end{aligned}
$$

Here, $E$ is the rank-5 bundle over $Y_{7}$ used in the construction of the sphere bundle, and $\mathcal{A}, \mathcal{F}$ are, respectively, the connection and curvature of the $\mathrm{SO}(5)$ gauge bundle, whereas $\bar{E}$ and $\overline{\mathcal{A}}, \overline{\mathcal{F}}$ are the corresponding extensions in the bulk $X_{8}$. In the last equation, we have used (4.13), and we recognise the topological term in the seven-dimensional supergravity Lagrangian (4.9). The choice of $\alpha=-1$, different from the case considered in section 4.1 is due to the different supersymmetry conventions used also in [65, 67].

It is known that the lowest-order correction to the equation of motion of the elevendimensional four-form is [24, 25]

$$
S_{1-\text { loop }}=-\alpha \frac{2 \pi}{\left(2 \pi \ell_{P}\right)^{3}} \int_{X_{12}} G \wedge \frac{p_{1}\left(X_{12}\right)^{2}-4 p_{2}\left(X_{12}\right)}{192} .
$$

In order to reduce this term to seven dimensions, we expand the Kaluza-Klein ansatz (4.6) to leading order in the derivatives, so that $\left.T X_{12}\right|_{X_{8}} \cong T X_{8} \oplus E$ and the spin connection is simply the sum of the two connections:

$$
\omega\left(X_{12}\right)^{A B}=\left(\begin{array}{cc}
\omega\left(X_{8}\right)^{a b} & 0 \\
0 & \mathfrak{g} \overline{\mathcal{A}}^{i j} \nabla^{m} K^{i j, n}
\end{array}\right),
$$

where $A, B$ are indices on $T X_{12}, a, b$ indices on $T X_{8}, \overline{\mathcal{A}}^{i j}$ are the components of the $\mathrm{SO}(5)$ gauge connection, $K^{i j}$ are Killing vectors generating the $\mathrm{SO}(5)$ isometries on $S^{4}$, and $m, n$ are indices on $T S^{4}$. Therefore, we write

$$
\begin{aligned}
& p_{1}\left(X_{12}\right)=p_{1}\left(X_{8}\right)+\mathfrak{g}^{2} p_{1}(\bar{E}), \\
& p_{2}\left(X_{12}\right)=p_{2}\left(X_{8}\right)+\mathfrak{g}^{2} p_{1}\left(X_{8}\right) \wedge p_{1}(\bar{E})+\mathfrak{g}^{4} p_{2}(\bar{E}) .
\end{aligned}
$$

\footnotetext{
${ }^{10}$ As a check, notice that this expression for $G$ gives, using (B.4)

$$
G=\frac{1}{8 \pi^{2}} \epsilon_{a_{1} a_{2} a_{3} a_{4} a_{5}} y^{a_{1}} D y^{a_{2}} \wedge D y^{a_{3}} \wedge D y^{a_{4}} \wedge D y^{a_{5}}+\ldots
$$

Setting $T_{i j}=\delta_{i j}$ in the full ansatz (4.7) gives $\Delta=1, U=-3$ and so we find the same expression at leading order.
} 
We can then integrate along the $S^{4}$ fiber, obtaining the one-loop correction to sevendimensional supergravity

$$
S_{1-\text { loop }}=-\alpha \frac{1}{24 \mathfrak{g}^{3} \ell_{P}^{3}} \int_{X_{8}}\left[-p_{2}\left(X_{8}\right)-\mathfrak{g}^{4} p_{2}(\bar{E})+\frac{1}{4}\left(\mathfrak{g}^{2} p_{1}(\bar{E})-p_{1}\left(X_{8}\right)\right)^{2}\right] .
$$

Altogether, using the quantization of the four-form flux and $\alpha=-1$, the topological terms in seven dimensions read

$$
\begin{aligned}
S_{7, \mathrm{top}}= & 2 \pi \int_{X_{8}}\left[\frac{N^{3}-N}{24} \mathfrak{g}^{4} p_{2}(\bar{E})\right. \\
& \left.+\frac{N}{48}\left(\mathfrak{g}^{4} p_{2}(\bar{E})-p_{2}\left(X_{8}\right)+\frac{1}{4}\left(\mathfrak{g}^{2} p_{1}(\bar{E})-p_{1}\left(X_{8}\right)\right)^{2}\right)\right] .
\end{aligned}
$$

As expected by AdS/CFT, this reproduces the structure of the $R$-symmetry anomaly of the six-dimensional $(2,0)$ SCFT obtained on the worldvolume of $N_{M 5} M 5$-branes, which reduces for $N_{M 5}=1$ to the anomaly of a free six-dimensional tensor multiplet (the second line).

Now consider the Pernici-Sezgin ansatz (4.18). We have

$$
\begin{aligned}
p_{1}\left(X_{8}\right) & =p_{1}\left(Y_{4}\right)+p_{1}\left(X_{4}\right), & p_{2}\left(X_{8}\right) & =p_{1}\left(Y_{4}\right) \wedge p_{1}\left(X_{4}\right), \\
p_{1}(\bar{E}) & =\frac{1}{\mathfrak{g}^{2}} p_{1}\left(X_{4}\right)+\frac{1}{\pi^{2}} F \wedge F, & p_{2}(\bar{E}) & =\frac{1}{\pi^{2} \mathfrak{g}^{2}} p_{1}\left(X_{4}\right) \wedge F \wedge F .
\end{aligned}
$$

and substitution leads to

$$
\begin{aligned}
S_{7, \text { top }} & =2 \pi \int_{X_{4}} p_{1}\left(X_{4}\right)\left(\frac{2 N_{M 5}^{3}-N_{M_{5}}}{6} \frac{\mathfrak{g}^{2}}{8 \pi^{2}} \int_{Y^{4}} F \wedge F-\frac{N_{M_{5}}}{48} \int_{Y_{4}} p_{1}\left(Y_{4}\right)\right) \\
& =-c s\left(\Sigma_{3}\right)\left(\frac{2 N_{M 5}^{3}-N_{M_{5}}}{3} \frac{\mathfrak{g}^{2}}{8 \pi^{2}} \int_{Y^{4}} F \wedge F-\frac{N_{M_{5}}}{24} \int_{Y_{4}} p_{1}\left(Y_{4}\right)\right) .
\end{aligned}
$$

These topological terms would appear in the semi-classical approximation of the gravity partition function. However, they are not well-defined even if $Y_{4}$ is a closed spin fourmanifold. In this case, we know that $\mathfrak{g}^{2} \int F^{2} \in 8 \pi^{2} \mathbb{Z}$, and $\int p_{1}\left(T Y_{4}\right)=3 \sigma\left(Y_{4}\right) \in 48 \mathbb{Z}$ (by Rokhlin's theorem), so we can write

$$
S_{7, \text { top }} \in \operatorname{cs}\left(\Sigma_{3}\right) \frac{2 N_{M 5}^{3}-7 N_{M_{5}}}{3} \mathbb{Z} .
$$

For compact $\Sigma_{3}$, the Chern-Simons invariant is well-defined modulo $2 \pi$, but the fraction is only an integer if $N_{M 5}$ is a multiple of 3 . This is consistent with the fact that the topological term in eleven dimensions is also not well-defined on its own [69].

The expression above also gives the subleading correction to (4.28) for the $\vartheta$ angle

$$
\vartheta=c s\left(\Sigma_{3}\right) \frac{2 N_{M 5}^{3}-N_{M_{5}}}{3}+o(N)
$$

From the discussion in section 2.4, the presence of the finite counterterm (2.36) guarantees that $\vartheta$ should be periodic with period $2 \pi$ even on a space with a boundary. However, 
the expression above is not periodic unless $N_{M 5}$ is a multiple of 3 (since $2 n^{3}+n \in 3 \mathbb{Z}$ for any integer $n$ ). Thus, we expect other subleading terms to contribute. At the same order as (4.33) there are corrections to the Einstein equations of motion in the form of $R^{4}$ terms [74], but these reduce in seven dimensions to $R^{3}$ terms and thus should not contribute to the $\vartheta$ angle in the further compactification following the Pernici-Sezgin ansatz.

The expression (4.41) has been obtained by reducing eleven-dimensional supergravity. However, it is also possible to obtain the higher-derivative correction to four-dimensional supergravity, as done in [10], and compare the results. Their expression for the Euclidean action including four-derivative corrections is

$$
S_{\mathrm{HD}}=S+\left(c_{1}+c_{2}\right) S_{W^{2}}+c_{2} S_{\mathrm{GB}}+16 \pi^{2} \mathrm{i} c_{3} \int p_{1}\left(Y_{4}\right)+16 \mathrm{i}\left(c_{3}+c_{4}\right) \mathfrak{g}^{2} \int F \wedge F,
$$

where $S$ is the two-derivative action $(2.1), S_{W^{2}}$ is a supersymmetrised version of the Weyl squared action, and $S_{\mathrm{GB}}$ is the Gauss-Bonnet action. This should be compared with the Wick-rotated version of the subleading action (4.39), which leads us to conclude that, for this compactification

$$
16 \pi^{2} c_{3}=-\operatorname{cs}\left(\Sigma_{3}\right) \frac{N}{24}, \quad c_{4}=0
$$

These results have been obtained by looking at the subleading corrections directly in the eleven-dimensional effective Lagrangian. However, in this approach it is difficult to justify whether one has accounted for all the relevant terms, as there are ambiguities due to potential field redefinitions. So, it is important to check them using independent methods, such as amplitudes [75].

\section{$5 \quad$ Field theory}

The AdS/CFT dictionary relates the on-shell gravity action in $d+1$ dimensions with the partition function of a field theory formulated on a $d$-dimensional manifold with the same geometric structure as the conformal boundary of the gravity solution. Which field theory should be considered depends on the knowledge of the full string/M-theory solution and hence on the choice of internal manifold.

As showed in section 4.1, we can embed solutions of four-dimensional minimal supergravity in eleven-dimensional supergravity by uplifting on seven-dimensional SasakiEinstein manifolds. In this case, there is no $\vartheta$ term in gravity and the on-shell action is real. The dual field theories are known for numerous choices of Sasaki-Einstein manifolds and are generically non-Abelian Chern-Simons-matter theories, for which the localization procedure is often known and the large $N$ limit of the partition function successfully compared with the gravity observable. This is the case that was discussed in [7].

On the other hand, in section 4.2 it was shown that the consistent truncation of eleven-dimensional supergravity on $\Sigma_{3} \times S^{4}$ leads to an additional $\vartheta$ term in the fourdimensional supergravity action (4.27), which makes the on-shell action generically complex in Euclidean signature. The dual field theory on $M_{3}$ is a $\mathcal{N}=2$ SCFT that is obtained by wrapping $N M 5$-branes on $M_{3} \times \Sigma_{3}$ and then looking at the IR compared to the energies associated with the compact $\Sigma_{3}$ [71]. It is the IR limit of a twisted compactification of the $A_{N-1}$ six-dimensional $(2,0)$ theory: the $\mathrm{SO}(3) \subset \mathrm{SO}(5)$ twisting in the gravity ansatz (4.18) 
corresponds in the dual field theory to the topological twist by the $\mathrm{SO}(3)_{R} \subset \mathrm{SO}(5)_{R}$, which indeed leaves a commutant $\mathrm{SO}(2)_{R} \cong \mathrm{U}(1)_{R}$ symmetry dual to the gauge field in fourdimensional supergravity. This $R$-symmetry is the one used to couple to the supergravity background necessary to formulate the theory on a curved $M_{3}$. The investigation of the resulting field theory $T_{N}\left[\Sigma_{3}\right]$ goes under the name of $3 d-3 d$ correspondence, and one of its crucial conjectures is that $T_{N}\left[\Sigma_{3}\right]$ should only depend on the topology of $\Sigma_{3}$ [27-29].

The construction of $T_{N}\left[\Sigma_{3}\right]$ has not yet been fully untangled, one of the issues being whether the vacua of $T_{N}\left[\Sigma_{3}\right]$ account for all the $\operatorname{PSL}(N ; \mathbb{C})$ flat connections on $\Sigma_{3}$ or only a subset thereof [76]. ${ }^{11}$ Here we are interested in the case where $\Sigma_{3}$ is hyperbolic. From the supergravity viewpoint, this is necessary in the ansatz (4.18), as otherwise the resulting consistent truncation does not preserve supersymmetry or have an AdS vacuum [72].

Generically, the large $N$ limit of the free energy of the field theory is computed using a saddle point approximation, and the dominant saddles contributing to the evaluation could lead to a complex value for the free energy. This is known to be the case for partition functions corresponding to twisted indices: complex saddle points contribute in conjugate pairs resulting in an oscillating twisted index (there is a growing literature on the subject, see e.g. [82-90]). Here we have showed that, at least for theories arising from $M 5$-branes, it is also true for the partition function on a generic fibred background. In fact, another crucial result of the $3 d-3 d$ correspondence is that the supersymmetric partition function of $T_{N}\left[\Sigma_{3}\right]$ on various spaces is captured by the partition function of $\operatorname{SL}(N, \mathbb{C})$ complex Chern-Simons theory on $\Sigma_{3}$ at an appropriate level (see [91-94] for a derivation). Therefore, combining the expectations from AdS/CFT and $3 d-3 d$ correspondence provides us with a conjecture for the large $N$ limit for the partition function of complex Chern-Simons theory as well.

To view concretely this limit, we first recall that Mostow's rigidity theorem guarantees that the hyperbolic metric on $\Sigma_{3}$ is unique, so $\operatorname{Vol}\left(\Sigma_{3}\right)$ is a topological invariant (in fact a homotopy invariant). The Chern-Simons invariant of the spin connection of the hyperbolic metric (4.20) is another topological invariant, which naturally combines with the hyperbolic volume in an invariant which is referred to as complex hyperbolic volume [95, 96]. More precisely, for a three-manifold both the dreibein and the spin connection can be seen as $\mathfrak{s o}(3)$ connections and we can introduce the $\mathfrak{s l}(2, \mathbb{C})$ geometric connection $\mathcal{A}^{\text {geom }}=\omega+$ ie and its complex conjugate $\mathcal{A}^{\overline{\mathrm{geom}}}$. This connection defines the complexified hyperbolic volume via the following relation

$$
\begin{aligned}
c s\left[\mathcal{A}^{\text {geom }}\right] & \equiv \frac{1}{8 \pi} \int_{\Sigma_{3}} \operatorname{tr}_{2}\left(\mathcal{A}^{\text {geom }} \wedge \mathrm{d} \mathcal{A}^{\text {geom }}+\frac{2}{3} \mathcal{A}^{\text {geom }} \wedge \mathcal{A}^{\text {geom }} \wedge \mathcal{A}^{\text {geom }}\right) \\
& =\frac{\mathrm{i}}{4 \pi}\left(\operatorname{Vol}\left(\Sigma_{3}\right)-\pi \mathrm{i} c s\left(\Sigma_{3}\right)\right) \\
& \equiv \frac{\mathrm{i}}{4 \pi} \operatorname{Vol}_{\mathbb{C}}\left(\Sigma_{3}\right), \\
\operatorname{cs}\left[\mathcal{A}^{\overline{\mathrm{geom}}}\right] & =-\frac{\mathrm{i}}{4 \pi} \operatorname{Vol}_{\mathbb{C}}\left(\Sigma_{3}\right)^{*} .
\end{aligned}
$$

\footnotetext{
${ }^{11}$ It is surely true that the structure of the theory is very different depending on whether $\Sigma_{3}$ is hyperbolic or not. In the latter case, if $\Sigma_{3}$ is a Seifert manifold the resulting field theory enjoys an additional $\mathrm{U}(1)_{f}$ symmetry associated with the circle action of the Seifert fibration (see e.g. [77-81] for the construction).
} 
First, observe that these are precisely the combination appearing in (2.55) after substitution of (4.28), so that we write

$$
\begin{aligned}
I^{\mathrm{IR}}= & \frac{4 \pi}{3 \Delta \psi^{2}} N^{3}\left[\sum_{\text {nuts }} \operatorname{Vol}_{\mathbb{C}}\left(\Sigma_{3}\right)^{*} \frac{\left(b_{1}+b_{2}\right)^{2}}{4 b_{1} b_{2}}-\sum_{\text {nuts }_{+}} \operatorname{Vol}_{\mathbb{C}}\left(\Sigma_{3}\right) \frac{\left(b_{1}-b_{2}\right)^{2}}{4 b_{1} b_{2}}\right. \\
& +\sum_{\text {bolts } \Sigma_{-}} \operatorname{Vol}_{\mathbb{C}}\left(\Sigma_{3}\right)^{*} \int_{\Sigma_{-}}\left(\frac{1}{2} c_{1}\left(T \Sigma_{-}\right)+\frac{1}{4} c_{1}\left(N \Sigma_{-}\right)\right) \\
& \left.+\sum_{\text {bolts } \Sigma_{+}} \operatorname{Vol}_{\mathbb{C}}\left(\Sigma_{3}\right) \int_{\Sigma_{+}}\left(\frac{1}{2} c_{1}\left(T \Sigma_{+}\right)-\frac{1}{4} c_{1}\left(N \Sigma_{+}\right)\right)\right] .
\end{aligned}
$$

We see that the complexified hyperbolic volume appears naturally in the expression for the IR contribution to the on-shell action. Provided a smooth gravity solution exists, this expression gives the contributions of the saddle points to the large $N$ limit of the partition function of the field theory on $M_{3} \cong \partial Y_{4}$ bounding $Y_{4}$. Of course, only some of them will be dominant. However, as already remarked, it is known that the field theory limit "sees" the contributions from various supergravity fillings, even in the case of Chern-Simons-matter field theories [2].

In order to be more concrete, we can consider various examples dual to those reviewed in section 3. To construct them, we shall need a few relations. First, given $h_{1}, h_{2} \in \mathfrak{p g r}(2)$ and denoting by $\rho_{N}$ the $N$-dimensional irreducible representation of $\mathfrak{p g l}(2)$, we have

$$
\operatorname{tr}\left[\rho_{N}\left(h_{1}\right) \rho_{N}\left(h_{2}\right)\right]=\frac{N^{3}-N}{6} \operatorname{tr}\left[h_{1} h_{2}\right] .
$$

Secondly, we shall need the large $N$ expansion of the Reidemeister-Ray-Singer torsion [20] ${ }^{12}$

$$
\begin{aligned}
\operatorname{Tor}_{\text {adj }}\left[\Sigma_{3}, \mathcal{A}^{\text {geom }}\right] & \rightarrow \exp \left(\frac{N^{3}}{3 \pi} \operatorname{Vol}\left(\Sigma_{3}\right)+\mathrm{i} \theta_{\Sigma_{3}, N}+\mathcal{O}(N)\right), \\
\operatorname{Tor}_{\text {adj }}\left[\Sigma_{3}, \mathcal{A}^{\overline{\text { geom }}}\right] & \rightarrow \exp \left(\frac{N^{3}}{3 \pi} \operatorname{Vol}\left(\Sigma_{3}\right)-\mathrm{i} \theta_{\Sigma_{3}, N}+\mathcal{O}(N)\right) .
\end{aligned}
$$

where $\theta_{\Sigma_{3}, N}$ is a real number. The large $N$ limit of the absolute value of the torsion is rigorously proved, whereas an expression for the phase factor has been conjectured in [6], where numerical evidence has been gathered. Here we shall find additional evidence in support of the conjecture

$$
\theta_{N, \Sigma_{3}}=-\frac{N^{3}}{3} \operatorname{cs}\left(\Sigma_{3}\right)+o\left(N^{3}\right) \quad \bmod 2 \pi
$$

to obtain

$$
\begin{aligned}
\operatorname{Tor}_{\text {adj }}\left[\Sigma_{3}, \mathcal{A}^{\text {geom }}\right] & \rightarrow \exp \left(\frac{N^{3}}{3 \pi} \operatorname{Vol}_{\mathbb{C}}\left(\Sigma_{3}\right)+\mathcal{O}(N)\right) \\
\operatorname{Tor}_{\text {adj }}\left[\Sigma_{3}, \mathcal{A}^{\overline{\text { geom }}}\right] & \rightarrow \exp \left(\frac{N^{3}}{3 \pi} \operatorname{Vol}_{\mathbb{C}}\left(\Sigma_{3}\right)^{*}+\mathcal{O}(N)\right) .
\end{aligned}
$$

\footnotetext{
${ }^{12}$ This is the analytic torsion of an associated vector bundle in a representation and twisted by a flat connection. For the case relevant to us, we restrict to the adjoint $\operatorname{PSL}(N, \mathbb{C})$ bundle and the flat connection will be either $\mathcal{A}^{\text {geom }}$ or $\mathcal{A}^{\overline{\text { geom }}}$.
} 
The solutions in section 3.2 have trivial fibration and there is a non-trivial flux of the gauge field at the boundary, so they are dual to field theories on supersymmetric backgrounds $M_{3} \cong S^{1} \times \Sigma_{g}$ where the $\mathrm{U}(1)_{R}$ gauge field is used to implement a topological twist and the resulting partition function is a supersymmetric index topologically twisted by the $R$-symmetry [97]. The requirement that $g>1$ for the supersymmetry of the gravity solutions corresponds to the fact that the large $N$ limit of the refined topologically twisted index on $S^{1} \times S^{2}$ vanishes (as showed using the $3 d-3 d$ correspondence in [22]). For $g>1$, the twisted index of $T_{N}\left[\Sigma_{3}\right]$ on $S^{1} \times \Sigma_{g}$ is related to the Reidemeister-Ray-Singer torsion of the irreducible flat $\mathrm{SL}(N, \mathbb{C})$ connections on $\Sigma_{3}$. The large $N$ behaviour of the resulting invariant is given by [20]

$$
\begin{aligned}
Z_{g, p=0}\left(T_{N}\left[\Sigma_{3}\right]\right) & \rightarrow N^{g-1} \mathbf{T o r}_{\text {adj }}\left[\Sigma_{3}, \mathcal{A}^{\text {geom }}\right]^{g-1}+N^{g-1} \mathbf{T o r}_{\text {adj }}\left[\Sigma_{3}, \mathcal{A}^{\overline{\text { geom }}}\right] \\
& \rightarrow \exp \left[-\frac{N^{3}}{3 \pi} \operatorname{Vol}_{\mathbb{C}}\left(\Sigma_{3}\right)(1-g)\right]+\exp \left[-\frac{N^{3}}{3 \pi} \operatorname{Vol}_{\mathbb{C}}\left(\Sigma_{3}\right)^{*}(1-g)\right],
\end{aligned}
$$

to leading order in $N$. The fact that modulus and phase combine into a complex topological invariant of $\Sigma_{3}$ was expected from the $3 d-3 d$ correspondence, since the partition function of $T_{N}\left[\Sigma_{3}\right]$ should only depend on the topology of $\Sigma_{3}$. The two contributions arise from saddle points corresponding to the geometric connection and its complex conjugate. As pointed out in [6], the two terms in the sum can be explained by identifying each with one of the two limiting solutions in (3.14), or equivalently the two solutions (3.26). Having done this, we obtain

$$
Z_{g, p=0}\left(T_{N}\left[\Sigma_{3}\right]\right)=\mathrm{e}^{-I\left[\text { Bolt }_{-}\right]}+\mathrm{e}^{-I\left[\text { Bolt }_{+}\right]},
$$

consistently with the expectations of AdS/CFT (we have set $\Delta \psi=2 \pi$ since both solutions are spin).

This can be easily generalised to the case with non-trivial fibration. The partition function of $T_{N}\left[\Sigma_{3}\right]$ on the space $\mathcal{M}_{g, p \in 2 \mathbb{Z}_{>0}}$ preserving two real supercharges can again be related to the Reidemeister-Ray-Singer torsion and the large $N$ of the resulting invariant computed [20]. In this case, the authors found a single saddle point that contribute to the evaluation, corresponding to the flat connection $\mathcal{A}^{\overline{\text { geom }}}$ :

$$
\begin{aligned}
Z_{g, p \in 2 \mathbb{Z}_{>0}}\left(T_{N}\left[\Sigma_{3}\right]\right) & \rightarrow N^{g-1} \exp \left(2 p \mathrm{i} c s\left[\rho_{N}\left(\mathcal{A}^{\overline{\mathrm{geom}}}\right)\right]\right) \operatorname{Tor}_{\mathrm{adj}}\left[\Sigma_{3}, \mathcal{A}^{\overline{\mathrm{geom}}}\right]^{g-1} \\
& \rightarrow \exp \left[-\frac{N^{3}}{3 \pi} \operatorname{Vol}_{\mathbb{C}}\left(\Sigma_{3}\right)^{*}\left(1-g-\frac{p}{4}\right)\right]
\end{aligned}
$$

The dual gravity solution is the $\frac{1}{4}$-BPS solution with topology $\mathcal{O}(-p) \rightarrow \Sigma_{g}$ with action (3.26), which is spin since we have chosen even $p$. So we write

$$
Z_{g, p \in 2 \mathbb{Z}_{>0}}\left(T_{N}\left[\Sigma_{3}\right]\right) \rightarrow \mathrm{e}^{-I^{\mathrm{IR}}\left[\mathrm{Bolt}_{+}\right]} .
$$

The existence of a unique saddle point is due to the fact that the family Bolt _ has larger on-shell action for $p>0$, whereas when $p=0$ the two solutions have the same on-shell action and thus have the same claim to represent a saddle point of the gravitational path integral. Furthermore, notice that the matching with the field theory supersymmetric 
partition function doesn't require $I^{\mathrm{UV}}$ (3.27), thus providing further evidence in favour of the necessity of the finite counterterm (2.36).

Finally, another supersymmetric background important for the $3 d-3 d$ correspondence is the $\mathrm{U}(1) \times \mathrm{U}(1)$ squashed three-sphere, which preserves two supercharges (3.8). The partition function of $T_{N}\left[\Sigma_{3}\right]$ on this background should be equal to the partition function of $\operatorname{SL}(N, \mathbb{C})$ on $\Sigma_{3}$ with parameters $\hbar$ and $\widetilde{\hbar}$ related to the squashing

$$
Z_{b}\left(T_{N}\left[\Sigma_{3}\right]\right)=Z_{\Sigma_{3}}\left(C S ; \hbar=2 \pi \mathrm{i} b^{2}, \widetilde{\hbar}=2 \pi \mathrm{i} b^{-2}\right) .
$$

The observable on the right-hand side is hard to compute. However, it was shown in [17] that the non-perturbative effects of order $\mathrm{e}^{-4 \pi^{2} / \hbar}$ are subleading in $N$, at least for the absolute value. If we can ignore the non-perturbative corrections, then $Z_{\Sigma_{3}}(C S)$ has the same asymptotic expansion in $\hbar$ as the perturbative expansion of the $\operatorname{SL}(N, \mathbb{C})$ ChernSimons partition function around the saddle point $\mathcal{A}^{\overline{\text { geom }}}$ :

$$
\begin{aligned}
Z_{\Sigma_{3}}(C S ; \hbar, \tilde{\hbar}) & \sim Z_{\Sigma_{3}}^{\text {pert }}\left(C S ; \hbar, \tilde{\hbar} ; \mathcal{A}^{\overline{\mathrm{geom}}}\right) \\
& =\exp \left(\frac{1}{\hbar} S_{0}^{\mathcal{A}^{\overline{\mathrm{geom}}}}-\frac{3}{2} \log \hbar+S_{1}^{\mathcal{A}^{\overline{\mathrm{geom}}}}+\hbar S_{2}^{\mathcal{A}^{\overline{\mathrm{geom}}}}+\cdots+\hbar^{n-1} S_{n}^{\mathcal{A}^{\overline{\mathrm{geom}}}}+\ldots\right)
\end{aligned}
$$

with

$$
\begin{aligned}
& S_{0}^{\mathcal{A}^{\overline{\mathrm{geom}}}}=4 \pi c s\left[\rho_{N}\left(\mathcal{A}^{\overline{\mathrm{geom}}}\right)\right] \sim-\mathrm{i} \frac{N^{3}}{6} \operatorname{Vol}_{\mathbb{C}}\left(\Sigma_{3}\right)^{*}+o\left(N^{3}\right), \\
& S_{1}^{\mathcal{A}^{\overline{\mathrm{geom}}}}=-\frac{1}{2} \log \mathbf{T o r}_{\mathrm{adj}}\left[\Sigma_{3}, \mathcal{A}^{\overline{\mathrm{geom}}}\right] \sim-\frac{N^{3}}{6 \pi} \operatorname{Vol}_{\mathbb{C}}\left(\Sigma_{3}\right)^{*}+o\left(N^{3}\right) .
\end{aligned}
$$

The expression for $S_{n}$ with $n \geq 2$ are not proved rigorously. However, the authors of [17] found numerical evidence in support of the following conjecture

$$
\begin{aligned}
\lim _{N \rightarrow \infty} \frac{1}{N^{3}} \operatorname{Im}\left[S_{2}^{\left.\mathcal{A}^{\overline{\mathrm{geom}}}\right]}\right. & =\frac{1}{24 \pi^{2}} \operatorname{Vol}\left(\Sigma_{3}\right), \\
\lim _{N \rightarrow \infty} \frac{1}{N^{3}} \operatorname{Im}\left[S_{2 j}^{\left.\mathcal{A}^{\overline{\mathrm{gom}}}\right]}\right. & =\lim _{N \rightarrow \infty} \frac{1}{N^{3}} \operatorname{Re}\left[S_{2 j-1}^{\mathcal{A}^{\overline{\mathrm{geom}}}}\right]=0 . \\
\lim _{N \rightarrow \infty} \frac{1}{N^{3}} S_{2}^{\mathcal{A}^{\overline{\mathrm{geom}}}} & =\frac{\mathrm{i}}{24 \pi^{2}} \operatorname{Vol}_{\mathbb{C}}\left(\Sigma_{3}\right)^{*}, \\
\lim _{N \rightarrow \infty} \frac{1}{N^{3}} S_{n}^{\mathcal{A}^{\overline{\mathrm{geom}}}} & =0 \quad \bmod 2 \pi \mathrm{i} \quad n \geq 3 .
\end{aligned}
$$

According to this conjecture, we would find

$$
Z_{b}\left(T_{N}\left[\Sigma_{3}\right]\right) \rightarrow \exp \left[-\frac{N^{3}}{3 \pi} \frac{1}{4}\left(b+\frac{1}{b}\right)^{2} \operatorname{Vol}_{\mathbb{C}}\left(\Sigma_{3}\right)^{*}\right]
$$

The dual solution is $\mathrm{AdS}_{4}^{(b)}$, meaning $\mathrm{AdS}_{4}$ with an instanton considered in section 3.1, with IR action (3.3), and indeed

$$
Z_{b}\left(T_{N}\left[\Sigma_{3}\right]\right) \rightarrow \mathrm{e}^{-I^{\mathrm{IR}}\left[\operatorname{AdS}_{4}^{(b)}\right]}
$$

Again the presence of a single saddle point corresponds to the gravity solution with larger on-shell action, and the absence of $I^{\mathrm{UV}}$ (3.12) supports the finite counterterm (2.36). 
As far as we know, this list exhausts the supersymmetric backgrounds for which the large $N$ limit of the $3 d-3 d$ correspondence has been studied. However, the examples considered in section 3 allow us to immediately conjecture the values of additional observables in the large $N$ limit. For instance, (3.26) shows that the large $N$ limit of the partition function of $T_{N}\left[\Sigma_{3}\right]$ on squashed $\mathcal{M}_{g, p}$ should not depend on the squashing parameter, consistently with the standard analysis of [9]. Similarly, the analysis of section 3.5 gives an expectation for the value of the large $N$ limit of the partition function of $T_{N}\left[\Sigma_{3}\right]$ on squashed Lens spaces, as studied in [94]. The interpretation of these partition functions is not obvious from the $3 d-3 d$ correspondence.

We should also point out that the supergravity ansatz made in order to construct the four-dimensional solutions holographically dual to $T_{N}\left[\Sigma_{3}\right]$ requires $\Sigma_{3}$ to be compact. However, it is also possible to construct a field theory $T_{N}\left[\Sigma_{3}\right]$ for cusped $\Sigma_{3}$, meaning that it is non-compact but with finite volume. In this case, it is more difficult to define a ChernSimons invariant because it is more difficult to find a section of the frame bundle. It is still possible to define an analogous invariant [98], in which case the complex hyperbolic volume is defined modulo $i \pi^{2}$ and still related to the integral of a $\mathfrak{s l}(2, \mathbb{C})$-valued geometric connection [96]. This invariant, though, also includes contributions from the cusps, which is not clear how to introduce in the supergravity setup.

\section{Acknowledgments}

I am grateful to James Sparks for very helpful discussions and comments on the draft. I have also benefited from conversations with Francesco Benini, Davide Cassani, Jerome Gauntlett, Chiung Hwang, Paul Richmond, Luigi Tizzano, David Tong, and Carl Turner. I would also like to thank the anonymous referee for their helpful suggestions to improve the manuscript. My work has been supported by the Simons Foundation, by the STFC consolidated grant ST/T000694/1, and by the ERC Consolidator Grant N. 681908 "Quantum black holes: A macroscopic window into the microstructure of gravity." I also gratefully acknowledge support and hospitality from the Galileo Galilei Institute.

\section{A Chern-Simons conventions}

Let $A=A^{\alpha} T^{\alpha}$ be a $\mathfrak{g}$ connection, and $T^{\alpha}$ the basis of $\mathfrak{g}$, with the following normalizations

$$
\begin{aligned}
\operatorname{tr}_{\mathbf{r}}\left(T^{\alpha} T^{\beta}\right) & =C(\mathbf{r}) \delta^{\alpha \beta}, \quad\left[T^{\alpha}, T^{\beta}\right]=f^{\alpha \beta \gamma} T^{\gamma}, \\
F & =\mathrm{d} A+A \wedge A=\left(\mathrm{d} A^{\alpha}+\frac{1}{2} f^{\alpha \beta \gamma} A^{\beta} \wedge A^{\gamma}\right) T^{\alpha},
\end{aligned}
$$

where $C(\mathbf{r})$ is (minus) the index of the representation $\mathbf{r}$. The Chern-Simons 3 -form is defined by

$$
\begin{aligned}
Q_{3}[A ; \mathfrak{g}, \mathbf{r}] & \equiv \operatorname{tr}_{\mathbf{r}}\left(A \wedge \mathrm{d} A+\frac{2}{3} A \wedge A \wedge A\right) \\
& =C(\mathbf{r})\left(A^{\alpha} \wedge \mathrm{d} A^{\alpha}+\frac{1}{3} f^{\alpha \beta \gamma} A^{\alpha} \wedge A^{\beta} \wedge A^{\gamma}\right)
\end{aligned}
$$

It has the property that $\mathrm{d} Q_{3}[A ; \mathfrak{g}, \mathbf{r}]=\operatorname{tr}_{\mathbf{r}}(F \wedge F)$. 
Now extend the definition to a complexified gauge group $G_{\mathbb{C}}$ with algebra $\mathfrak{g}_{\mathbb{C}}$ for which $G$ is the compact real form. Write $\mathcal{A} \equiv A+\mathrm{i} B$ for the $\mathfrak{g}_{\mathbb{C}}$-valued connection with $A, B$ $\mathfrak{g}$-valued connections. The curvature is

$$
\mathcal{F}=F_{A}+\mathrm{i} D_{A} B-B \wedge B
$$

where

$$
\left(D_{A} B\right)^{\alpha} \equiv \mathrm{d} B^{\alpha}+f^{\alpha \beta \gamma} A^{\beta} \wedge B^{\gamma} .
$$

The Chern-Simons form for the complex connection is

$$
\begin{aligned}
Q_{3}\left[\mathcal{A} ; \mathfrak{g}_{\mathbb{C}}, \mathbf{r}\right]= & \operatorname{tr}_{\mathbf{r}}\left(\mathcal{A} \wedge \mathrm{d} \mathcal{A}+\frac{2}{3} \mathcal{A} \wedge \mathcal{A} \wedge \mathcal{A}\right) \\
= & Q_{3}[A ; \mathfrak{g}, \mathbf{r}]-\operatorname{tr}\left(B \wedge D_{A} B\right)+2 \mathrm{i} \operatorname{tr}_{\mathbf{r}}\left(B \wedge F_{A}-\frac{1}{3} B \wedge B \wedge B\right) \\
& -i \mathrm{~d}\left(\operatorname{tr}_{\mathbf{r}} A \wedge B\right)
\end{aligned}
$$

The action of $G_{\mathbb{C}}$ Chern-Simons theory is

$$
\begin{aligned}
I & =\frac{k+\mathrm{i} s}{8 \pi} \int Q_{3}[\mathcal{A} ; \mathfrak{g}, \mathbf{r}]+\frac{k-\mathrm{i} s}{8 \pi} \int Q_{3}[\overline{\mathcal{A}} ; \mathfrak{g}, \mathbf{r}] \\
& =\frac{k}{4 \pi} \int\left(Q_{3}[A ; \mathfrak{g}, \mathbf{r}]-\operatorname{tr}\left(B \wedge D_{A} B\right)\right)-\frac{s}{2 \pi} \int \operatorname{tr}_{\mathbf{r}}\left(B \wedge F_{A}-\frac{1}{3} B \wedge B \wedge B\right) .
\end{aligned}
$$

Invariance under large gauge transformations requires $k \in \mathbb{Z}$, and $u$ is either real or purely imaginary in order to have a unitary theory [99].

We are interested in $\mathfrak{s o}(N)$ bundles. A connection of $\mathfrak{s o}(N)$ in the defining representation is concretely represented by an anti-symmetric matrix $A=A^{a b}$ with $A^{a b}=-A^{b a}$. Then we can define the Chern-Simons form (for $\mathfrak{s o}(N), C($ fund $)=1$ )

$$
Q_{3}[A ; \mathfrak{s o}(N), \text { fund }]=\operatorname{tr}\left(A \wedge \mathrm{d} A+\frac{2}{3} A \wedge A \wedge A\right) .
$$

A standard argument based on extension in the bulk then shows that the integral of $Q_{3}[A ; \mathfrak{s o}(N)$, fund $]$ on $\Sigma_{3}$ is well-defined modulo $16 \pi^{2}$ if we choose a spin structure on $\Sigma_{3}$, otherwise modulo $8 \pi^{2}$.

Over a three-manifold there is a special $\mathfrak{s o}(3)$ bundle, the frame bundle. The connection is the spin connection $\omega^{a b}$, and we can define the Chern-Simons form

$$
Q_{3}[\omega ; \mathfrak{s o}(3), \mathbf{3}] \equiv \operatorname{tr}_{\mathbf{3}}\left(\omega \wedge \mathrm{d} \omega+\frac{2}{3} \omega \wedge \omega \wedge \omega\right) .
$$

For a closed oriented 3-manifold with a choice of spin structure, this defines a topological invariant defined modulo $2 \pi$

$$
\operatorname{cs}\left(\Sigma_{3}\right) \equiv \frac{1}{8 \pi} \int_{\Sigma_{3}} Q_{3}[\omega ; \mathfrak{s o}(3), \mathbf{3}] \in \mathbb{R} / 2 \pi \mathbb{Z},
$$

which is well-defined modulo $2 \pi$. However, it is also possible to define a topological invariant without choosing the spin structure, using the Chern-Simons "at level one" (2.37)

$$
\frac{1}{4 \pi} \int_{\Sigma_{3}} Q_{3}[\omega ; \mathfrak{s o}(3), \mathbf{3}]
$$


In fact, for $\mathfrak{s o}(3)$ more is true, since both the dreibein and the spin connection can be seen as $\mathfrak{s o}(3)$ connections. Thus, we can dualize the index and write

$$
\widetilde{\omega}^{a} \equiv \frac{1}{2} \epsilon^{a b c} \omega^{b c}, \quad \omega^{a b}=\epsilon^{a b c} \widetilde{\omega}^{c} .
$$

dualizing the index. This allows us to construct a natural complex connection for $\mathfrak{g}_{\mathbb{C}}=$ $\mathfrak{s l}(2, \mathbb{C})$ :

$$
\mathcal{A}_{\text {geom }} \equiv \widetilde{\omega}^{a}+\mathrm{ie}^{a} .
$$

This is the geometric connection, whose curvature can be found applying (A.3)

$$
\mathcal{F}_{\text {geom }}^{a}=\widetilde{\rho}^{a}+\frac{1}{2} \epsilon^{a b c} e^{b} \wedge e^{c},
$$

where $\rho^{a b}$ is the curvature two-form. Substitution in (A.5) leads to

$$
\begin{aligned}
\int Q_{3}\left[\mathcal{A}_{\text {geom }} ; \mathfrak{s l}(2, \mathbb{C}), \mathbf{2}\right] & =\int Q_{3}[\omega ; \mathfrak{s u}(2), \mathbf{2}]+2 \mathrm{i} \int \operatorname{tr}_{\mathbf{2}}\left(\mathrm{e} \wedge \widetilde{\rho}-\frac{1}{3} \mathrm{e} \wedge \mathrm{e} \wedge \mathrm{e}\right) \\
& =\int Q_{3}[\omega ; \mathfrak{s u}(2), \mathbf{2}]-\frac{\mathrm{i}}{2} \int(R+2) \operatorname{vol}_{g}
\end{aligned}
$$

Changing the representation of $\mathfrak{s u}(2)$, we conclude that for a compact 3-manifold with a chosen spin structure

$$
\int_{\Sigma_{3}} Q_{3}\left[\mathcal{A}_{\text {geom }} ; \mathfrak{s l}(2, \mathbb{C}), \mathbf{2}\right]=2 \pi \operatorname{cs}\left(\Sigma_{3}\right)-\frac{\mathrm{i}}{2} \int_{\Sigma_{3}}(R+2) \operatorname{vol}_{g}
$$

Now choose a hyperbolic 3-manifold. By hyperbolic 3-manifold we mean a complete Riemannian three-dimensional manifold with constant sectional curvature -1 . All these manifolds can be obtained as quotients of $\mathbb{H}^{3} / \Gamma$, where $\Gamma$ is a Kleinian group, a discrete subgroup of $\operatorname{PSL}(2, \mathbb{C})$. We also restrict to the case of manifolds with finite volume. Mostow's rigidity theorem guarantees that for this class of manifolds the hyperbolic structure is uniquely determined by the homotopy type, so the volume is a topological invariant. Note that these manifolds (with finite volume) can be compact or non-compact. A non-compact hyperbolic manifold without boundary and finite volume is also called cusped.

For a hyperbolic manifold, (A.13) shows that the geometric connection is flat, and we can simplify (A.15) to

$$
\int_{\Sigma_{3}}\left(-\frac{\mathrm{i}}{2} Q_{3}\left[\mathcal{A}_{\text {geom }} ; \mathfrak{s l}(2, \mathbb{C}), \mathbf{2}\right]\right)=\operatorname{Vol}\left(\Sigma_{3}\right)-\pi \mathrm{i} c s\left(\Sigma_{3}\right) .
$$

The quantity

$$
\operatorname{Vol}_{\mathbb{C}}\left(\Sigma_{3}\right) \equiv \operatorname{Vol}\left(\Sigma_{3}\right)-\pi \mathrm{i} c s\left(\Sigma_{3}\right)=\int_{\Sigma_{3}}\left(-\frac{\mathrm{i}}{2} Q_{3}\left[\mathcal{A}_{\text {geom }} ; \mathfrak{s l}(2, \mathbb{C}), \mathbf{2}\right]\right)
$$

is the complex hyperbolic volume of the compact hyperbolic three-manifold [95, 96]. It is a topological invariant defined modulo i $2 \pi^{2}$ (having chosen the spin structure). Therefore, it is natural to write it as an exponential

$$
\exp \left(\frac{1}{\pi} \operatorname{Vol}_{\mathbb{C}}\left(\Sigma_{3}\right)\right)=\exp \left(\frac{1}{\pi} \operatorname{Vol}_{\mathbb{C}}\left(\Sigma_{3}\right)\right) \mathrm{e}^{-\mathrm{i} c s\left(\Sigma_{3}\right)} .
$$


We are also interested in non-compact hyperbolic three-manifold (that is, with cusps). For instance, most knot complements are non-compact. In this case, it not necessarily true that there is a global section of the frame bundle that can be used to compute the ChernSimons invariant using the formula (A.8) with $Q_{3}$. However, Meyerhoff [98] has showed that there is a way of defining a Chern-Simons invariant by defining a special singular frame, and adding the contribution from the singularities at the cusps:

$$
c s^{\mathrm{Me}}\left(\Sigma_{3}\right)=-\frac{1}{8 \pi} \int_{s\left(\Sigma_{3} \backslash L\right)} Q_{3}[\omega ; \mathfrak{s o}(3), \mathbf{3}]+\frac{1}{2} \sum_{K \in L} \tau(K) \bmod \pi,
$$

where $L$ is a link in $\Sigma_{3}$, with components $K, s: \Sigma_{3} \backslash L \rightarrow F\left(M_{3}\right)$ is a special singular frame, and $\tau(K)$ is the torsion of the singular curve $K$ in $L$. This can also be used to construct a complexification of the hyperbolic volume, by defining

$$
\operatorname{Vol}_{\mathbb{C}}^{\mathrm{Me}}\left(\Sigma_{3}\right) \equiv \operatorname{Vol}\left(\Sigma_{3}\right)-\pi \mathrm{i} c s^{\mathrm{Me}}\left(\Sigma_{3}\right)
$$

which is defined modulo $i \pi^{2}$. Just like in the case of compact hyperbolic manifolds, this Chern-Simons invariant can also be related to the integral of the $\mathfrak{s l}(2, \mathbb{C})$-valued geometric connection [96].

\section{B Bott-Cattaneo formula}

For an $\mathfrak{o}(N)$ vector bundle $E$ with curvature $F$ (represented by an antisymmetric matrix) we can define the Pontryagin classes

$$
p_{1} \equiv-\frac{1}{8 \pi^{2}} \operatorname{tr} F^{2}, \quad p_{2} \equiv \frac{1}{8(2 \pi)^{4}}\left[\left(\operatorname{tr} F^{2}\right)^{2}-2 \operatorname{tr} F^{4}\right] .
$$

This is in particular true of the tangent bundle.

Let $E \rightarrow B$ be a rank $2 m+1$ vector bundle over $B$. The fiber over $p \in B$ is a copy of $\mathbb{R}^{2 m+1}$ parametrized by $y^{a}, a=1, \ldots, 2 m+1$, equipped with a fiber metric. The sphere bundle $S(E)$ associated to $E$ is the bundle $S(E) \rightarrow B$ whose fiber over $p \in B$ is the unit sphere $S^{2 m} \subset E_{p} \cong \mathbb{R}^{2 m+1}$. This bundle has structure group $\mathrm{SO}(2 m+1)$ and the non-triviality is represented by the connection $A^{a b}$. This allows us to define the forms

$$
D y^{a} \equiv \mathrm{d} y^{a}+\mathfrak{g} A^{a b} y^{b}, \quad F^{a b} \equiv \mathrm{d} A^{a b}+\mathfrak{g} A^{a c} \wedge A^{c b} .
$$

The global angular form for $S(E)$ is the unique closed and gauge-invariant improvement of the sphere volume form. For $m=1,2$, it has the following form

$$
\begin{aligned}
\mathrm{E}_{2} \equiv & \frac{1}{8 \pi} \epsilon_{a_{1} a_{2} a_{3}} y^{a_{1}}\left[D y^{a_{2}} \wedge D y^{a_{3}}+\mathfrak{g} F^{a_{2} a_{3}}\right] \\
\mathrm{E}_{4} \equiv & \frac{1}{64 \pi^{2}} \epsilon_{a_{1} a_{2} a_{3} a_{4} a_{5}} y^{a_{1}}\left[D y^{a_{2}} \wedge D y^{a_{3}} \wedge D y^{a_{4}} \wedge D y^{a_{5}}+2 \mathfrak{g} F^{a_{2} a_{3}} \wedge D y^{a_{4}} \wedge D y^{a_{5}}\right. \\
& \left.+\mathfrak{g}^{2} F^{a_{2} a_{3}} \wedge F^{a_{4} a_{5}}\right] .
\end{aligned}
$$

Here, we have chosen a normalization such that the global angular form integrates to 1 along the fiber:

$$
\int_{S^{2 m}} \mathrm{E}_{2 m}=1
$$


The Bott-Cattaneo formula gives a relation between the integral of the global angular form along the fibers, and the characteristic classes of the $\mathrm{SO}(2 m+1)$ bundle over $B$ :

$$
\int_{S^{2 m}}\left(\mathrm{E}_{2 m}\right)^{2 s+2}=0, \quad \int_{S^{2 m}}\left(\mathrm{E}_{2 m}\right)^{2 s+1}=\frac{\mathfrak{g}^{2 m s}}{2^{2 s}}\left[p_{m}(E)\right]^{s} .
$$

Open Access. This article is distributed under the terms of the Creative Commons Attribution License (CC-BY 4.0), which permits any use, distribution and reproduction in any medium, provided the original author(s) and source are credited.

\section{References}

[1] D.Z. Freedman and A.K. Das, Gauge Internal Symmetry in Extended Supergravity, Nucl. Phys. B 120 (1977) 221 [inSPIRE].

[2] C. Toldo and B. Willett, Partition functions on 3d circle bundles and their gravity duals, JHEP 05 (2018) 116 [arXiv:1712.08861] [INSPIRE].

[3] O. Aharony, F. Benini, O. Mamroud and P. Milan, A gravity interpretation for the Bethe Ansatz expansion of the $\mathcal{N}=4$ SYM index, Phys. Rev. D 104 (2021) 086026 [arXiv:2104.13932] [INSPIRE].

[4] D.Z. Freedman and S.S. Pufu, The holography of F-maximization, JHEP 03 (2014) 135 [arXiv: 1302.7310] [INSPIRE].

[5] N. Bobev, A.M. Charles and V.S. Min, Euclidean black saddles and AdS 4 black holes, JHEP 10 (2020) 073 [arXiv: 2006. 01148] [inSPIRE].

[6] S. Choi, D. Gang and N. Kim, Black holes and large $N$ complex saddles in 3D-3D correspondence, JHEP 06 (2021) 078 [arXiv: 2012.10944] [INSPIRE].

[7] P. Benetti Genolini, J.M. Perez Ipiña and J. Sparks, Localization of the action in AdS/CFT, JHEP 10 (2019) 252 [arXiv:1906.11249] [InSPIRE].

[8] E. Witten, $\mathrm{SL}(2, \mathbb{Z})$ action on three-dimensional conformal field theories with Abelian symmetry, in From Fields to Strings: Circumnavigating Theoretical Physics: A Conference in Tribute to Ian Kogan, Oxford U.K. (2003), pg. 1173 [hep-th/0307041] [INSPIRE].

[9] C. Closset, T.T. Dumitrescu, G. Festuccia and Z. Komargodski, The Geometry of Supersymmetric Partition Functions, JHEP 01 (2014) 124 [arXiv:1309.5876] [InSPIRE].

[10] N. Bobev, A.M. Charles, K. Hristov and V. Reys, Higher-derivative supergravity, AdS $S_{4}$ holography, and black holes, JHEP 08 (2021) 173 [arXiv:2106.04581] [INSPIRE].

[11] P.B. Genolini and P. Richmond, Supersymmetry of higher-derivative supergravity in AdS4 holography, Phys. Rev. D 104 (2021) L061902 [arXiv:2107.04590] [INSPIRE].

[12] K. Hristov and V. Reys, Factorization of log-corrections in $A d S_{4} / C F T_{3}$ from supergravity localization, JHEP 12 (2021) 031 [arXiv:2107.12398] [INSPIRE].

[13] D. Cassani, J.P. Gauntlett, D. Martelli and J. Sparks, Thermodynamics of accelerating and supersymmetric AdS4 black holes, Phys. Rev. D 104 (2021) 086005 [arXiv:2106.05571] [INSPIRE].

[14] J.P. Gauntlett and O. Varela, Consistent Kaluza-Klein reductions for general supersymmetric AdS solutions, Phys. Rev. D 76 (2007) 126007 [arXiv:0707.2315] [INSPIRE]. 
[15] G. Larios and O. Varela, Minimal $D=4 \mathcal{N}=2$ supergravity from $D=11$ : An M-theory free lunch, JHEP 10 (2019) 251 [arXiv: 1907.11027] [INSPIRE].

[16] J.P. Gauntlett, O.A.P. Mac Conamhna, T. Mateos and D. Waldram, AdS spacetimes from wrapped M5 branes, JHEP 11 (2006) 053 [hep-th/0605146] [INSPIRE].

[17] D. Gang, N. Kim and S. Lee, Holography of 3d-3d correspondence at Large N, JHEP 04 (2015) 091 [arXiv:1409.6206] [inSPIRE].

[18] D. Gang, N. Kim, M. Romo and M. Yamazaki, Aspects of Defects in 3d-3d Correspondence, JHEP 10 (2016) 062 [arXiv:1510.05011] [INSPIRE].

[19] D. Gang and N. Kim, Large $N$ twisted partition functions in 3d-3d correspondence and Holography, Phys. Rev. D 99 (2019) 021901 [arXiv: 1808.02797] [INSPIRE].

[20] D. Gang, N. Kim and L.A. Pando Zayas, Precision Microstate Counting for the Entropy of Wrapped M5-branes, JHEP 03 (2020) 164 [arXiv: 1905.01559] [INSPIRE].

[21] N. Bobev and P.M. Crichigno, Universal spinning black holes and theories of class $\mathcal{R}$, JHEP 12 (2019) 054 [arXiv: 1909.05873] [INSPIRE].

[22] F. Benini, D. Gang and L.A. Pando Zayas, Rotating Black Hole Entropy from M5 Branes, JHEP 03 (2020) 057 [arXiv: 1909.11612] [InSPIRE].

[23] N. Bobev, A.M. Charles, D. Gang, K. Hristov and V. Reys, Higher-derivative supergravity, wrapped M5-branes, and theories of class $\mathcal{R}$, JHEP 04 (2021) 058 [arXiv:2011.05971] [INSPIRE].

[24] C. Vafa and E. Witten, A One loop test of string duality, Nucl. Phys. B 447 (1995) 261 [hep-th/9505053] [INSPIRE].

[25] M.J. Duff, J.T. Liu and R. Minasian, Eleven-dimensional origin of string-string duality: A One loop test, Nucl. Phys. B 452 (1995) 261 [hep-th/9506126] [InSPIRE].

[26] Y. Terashima and M. Yamazaki, SL $(2, \mathbb{R})$ Chern-Simons, Liouville, and Gauge Theory on Duality Walls, JHEP 08 (2011) 135 [arXiv:1103.5748] [INSPIRE].

[27] T. Dimofte, D. Gaiotto and S. Gukov, Gauge Theories Labelled by Three-Manifolds, Commun. Math. Phys. 325 (2014) 367 [arXiv:1108.4389] [INSPIRE].

[28] S. Cecotti, C. Cordova and C. Vafa, Braids, Walls, and Mirrors, arXiv:1110.2115 [INSPIRE].

[29] T. Dimofte, D. Gaiotto and S. Gukov, 3-Manifolds and 3d Indices, Adv. Theor. Math. Phys. 17 (2013) 975 [arXiv: 1112.5179] [INSPIRE].

[30] M. Dunajski, J.B. Gutowski, W.A. Sabra and P. Tod, Cosmological Einstein-Maxwell Instantons and Euclidean Supersymmetry: Beyond Self-Duality, JHEP 03 (2011) 131 [arXiv: 1012.1326] [INSPIRE].

[31] D. Martelli, A. Passias and J. Sparks, The supersymmetric NUTs and bolts of holography, Nucl. Phys. B 876 (2013) 810 [arXiv:1212.4618] [InSPIRE].

[32] R. Emparan, C.V. Johnson and R.C. Myers, Surface terms as counterterms in the AdS/CFT correspondence, Phys. Rev. D 60 (1999) 104001 [hep-th/9903238] [InSPIRE].

[33] K. Skenderis, Lecture notes on holographic renormalization, Class. Quant. Grav. 19 (2002) 5849 [hep-th/0209067] [INSPIRE].

[34] P. Benetti Genolini, D. Cassani, D. Martelli and J. Sparks, Holographic renormalization and supersymmetry, JHEP 02 (2017) 132 [arXiv:1612.06761] [INSPIRE]. 
[35] C. Closset, T.T. Dumitrescu, G. Festuccia and Z. Komargodski, Supersymmetric Field Theories on Three-Manifolds, JHEP 05 (2013) 017 [arXiv:1212.3388] [INSPIRE].

[36] C. Klare, A. Tomasiello and A. Zaffaroni, Supersymmetry on Curved Spaces and Holography, JHEP 08 (2012) 061 [arXiv: 1205.1062] [INSPIRE].

[37] C. Closset and H. Kim, Three-dimensional $\mathscr{N}=2$ supersymmetric gauge theories and partition functions on Seifert manifolds: A review, Int. J. Mod. Phys. A 34 (2019) 1930011 [arXiv: 1908.08875] [INSPIRE].

[38] D. Marolf and S.F. Ross, Boundary Conditions and New Dualities: Vector Fields in AdS/CFT, JHEP 11 (2006) 085 [hep-th/0606113] [INSPIRE].

[39] G.W. Gibbons and S.W. Hawking, Classification of Gravitational Instanton Symmetries, Commun. Math. Phys. 66 (1979) 291 [INSPIRE].

[40] D.Z. Freedman, K. Pilch, S.S. Pufu and N.P. Warner, Boundary Terms and Three-Point Functions: An AdS/CFT Puzzle Resolved, JHEP 06 (2017) 053 [arXiv:1611.01888] [INSPIRE].

[41] J.P. Gauntlett and C. Rosen, Susy $Q$ and spatially modulated deformations of ABJM theory, JHEP 10 (2018) 066 [arXiv:1808.02488] [INSPIRE].

[42] P. Benetti Genolini, M. Grinberg and P. Richmond, Boundary conditions in topological $A d S_{4} / C F T_{3}, J H E P 02$ (2021) 156 [arXiv:2010.15828] [INSPIRE].

[43] E. Witten, On S duality in Abelian gauge theory, Selecta Math. 1 (1995) 383 [hep-th/9505186] [INSPIRE].

[44] S.W. Hawking and S.F. Ross, Duality between electric and magnetic black holes, Phys. Rev. $D 52$ (1995) 5865 [hep-th/9504019] [INSPIRE].

[45] R.G. Leigh and A.C. Petkou, SL $(2, \mathbb{Z})$ action on three-dimensional CFTs and holography, JHEP 12 (2003) 020 [hep-th/0309177] [INSPIRE].

[46] R. Zucchini, Four-dimensional Abelian duality and $\mathrm{SL}(2, \mathbb{Z})$ action in three-dimensional conformal field theory, Adv. Theor. Math. Phys. 8 (2004) 895 [hep-th/0311143] [InSPIRE].

[47] H.-U. Yee, A Note on AdS/CFT dual of $\mathrm{SL}(2, \mathbb{Z})$ action on $3-D$ conformal field theories with U(1) symmetry, Phys. Lett. B 598 (2004) 139 [hep-th/0402115] [INSPIRE].

[48] M. Roček and P. van Nieuwenhuizen, $N \geq 2$ Supersymmetric Chern-Simons Terms as $d=3$ Extended Conformal Supergravity, Class. Quant. Grav. 3 (1986) 43 [INSPIRE].

[49] C. Closset, T.T. Dumitrescu, G. Festuccia, Z. Komargodski and N. Seiberg, Comments on Chern-Simons Contact Terms in Three Dimensions, JHEP 09 (2012) 091 [arXiv: 1206.5218] [INSPIRE].

[50] D. Martelli, A. Passias and J. Sparks, The gravity dual of supersymmetric gauge theories on a squashed three-sphere, Nucl. Phys. B 864 (2012) 840 [arXiv:1110.6400] [InSPIRE].

[51] D. Farquet, J. Lorenzen, D. Martelli and J. Sparks, Gravity duals of supersymmetric gauge theories on three-manifolds, JHEP 08 (2016) 080 [arXiv: 1404.0268] [INSPIRE].

[52] N. Hama, K. Hosomichi and S. Lee, SUSY Gauge Theories on Squashed Three-Spheres, JHEP 05 (2011) 014 [arXiv:1102.4716] [INSPIRE].

[53] L.F. Alday, D. Martelli, P. Richmond and J. Sparks, Localization on Three-Manifolds, JHEP 10 (2013) 095 [arXiv: 1307.6848] [inSPIRE]. 
[54] L.J. Romans, Supersymmetric, cold and lukewarm black holes in cosmological Einstein-Maxwell theory, Nucl. Phys. B 383 (1992) 395 [hep-th/9203018] [INSPIRE].

[55] D.R. Brill, J. Louko and P. Peldan, Thermodynamics of $(3+1)$-dimensional black holes with toroidal or higher genus horizons, Phys. Rev. D 56 (1997) 3600 [gr-qc/9705012] [INSPIRE].

[56] M.M. Caldarelli and D. Klemm, Supersymmetry of Anti-de Sitter black holes, Nucl. Phys. B 545 (1999) 434 [hep-th/9808097] [INSPIRE].

[57] D. Martelli and J. Sparks, The gravity dual of supersymmetric gauge theories on a biaxially squashed three-sphere, Nucl. Phys. B 866 (2013) 72 [arXiv:1111.6930] [InSPIRE].

[58] M. Gabella, D. Martelli, A. Passias and J. Sparks, $\mathcal{N}=2$ supersymmetric AdS $S_{4}$ solutions of M-theory, Commun. Math. Phys. 325 (2014) 487 [arXiv:1207.3082] [INSPIRE].

[59] A. Guarino, D.L. Jafferis and O. Varela, String Theory Origin of Dyonic $N=8$ Supergravity and Its Chern-Simons Duals, Phys. Rev. Lett. 115 (2015) 091601 [arXiv:1504.08009] [INSPIRE].

[60] O. Varela, Minimal D = 4 truncations of type IIA, JHEP 11 (2019) 009 [arXiv:1908.00535] [INSPIRE].

[61] M. Fluder and J. Sparks, D2-brane Chern-Simons theories: F-maximization = a-maximization, JHEP 01 (2016) 048 [arXiv:1507.05817] [INSPIRE].

[62] M.J. Duff, B.E.W. Nilsson, C.N. Pope and N.P. Warner, On the Consistency of the Kaluza-Klein Ansatz, Phys. Lett. B 149 (1984) 90 [INSPIRE].

[63] N. Benishti, D. Rodriguez-Gomez and J. Sparks, Baryonic symmetries and M5 branes in the $A d S_{4} / C F T_{3}$ correspondence, JHEP 07 (2010) 024 [arXiv: 1004.2045] [INSPIRE].

[64] H. Nastase, D. Vaman and P. van Nieuwenhuizen, Consistent nonlinear $K$ K reduction of $11-D$ supergravity on $A d S_{7} \times S^{4}$ and selfduality in odd dimensions, Phys. Lett. $B 469$ (1999) 96 [hep-th/9905075] [INSPIRE].

[65] H. Nastase, D. Vaman and P. van Nieuwenhuizen, Consistency of the $A d S_{7} \times S^{4}$ reduction and the origin of selfduality in odd dimensions, Nucl. Phys. B 581 (2000) 179 [hep-th/9911238] [INSPIRE].

[66] H. Lü and C.N. Pope, Exact embedding of $N=1, D=7$ gauged supergravity in $D=11$, Phys. Lett. B 467 (1999) 67 [hep-th/9906168] [INSPIRE].

[67] M. Cvetič, H. Lü, C.N. Pope, A. Sadrzadeh and T.A. Tran, $S^{3}$ and $S^{4}$ reductions of type IIA supergravity, Nucl. Phys. B 590 (2000) 233 [hep-th/0005137] [INSPIRE].

[68] M. Pernici, K. Pilch and P. van Nieuwenhuizen, Gauged Maximally Extended Supergravity in Seven-dimensions, Phys. Lett. B 143 (1984) 103 [InSPIRE].

[69] E. Witten, On flux quantization in M-theory and the effective action, J. Geom. Phys. 22 (1997) 1 [hep-th/9609122] [INSPIRE].

[70] M. Pernici and E. Sezgin, Spontaneous Compactification of Seven-dimensional Supergravity Theories, Class. Quant. Grav. 2 (1985) 673 [INSPIRE].

[71] J.P. Gauntlett, N. Kim and D. Waldram, M Five-branes wrapped on supersymmetric cycles, Phys. Rev. D 63 (2001) 126001 [hep-th/0012195] [INSPIRE].

[72] A. Donos, J.P. Gauntlett, N. Kim and O. Varela, Wrapped M5-branes, consistent truncations and AdS/CMT, JHEP 12 (2010) 003 [arXiv:1009.3805] [INSPIRE]. 
[73] J.A. Harvey, R. Minasian and G.W. Moore, NonAbelian tensor multiplet anomalies, JHEP 09 (1998) 004 [hep-th/9808060] [INSPIRE].

[74] A.A. Tseytlin, $R^{4}$ terms in 11 dimensions and conformal anomaly of (2,0) theory, Nucl. Phys. B 584 (2000) 233 [hep-th/0005072] [INSPIRE].

[75] S.M. Chester, S.S. Pufu and X. Yin, The M-theory S-matrix From ABJM: Beyond 11D Supergravity, JHEP 08 (2018) 115 [arXiv: 1804.00949] [INSPIRE].

[76] H.-J. Chung, T. Dimofte, S. Gukov and P. Sułkowski, 3d-3d Correspondence Revisited, JHEP 04 (2016) 140 [arXiv: 1405.3663] [inSPIRE].

[77] S. Gukov and D. Pei, Equivariant Verlinde formula from fivebranes and vortices, Commun. Math. Phys. 355 (2017) 1 [arXiv:1501.01310] [InSPIRE].

[78] D. Pei and K. Ye, A 3d-3d appetizer, JHEP 11 (2016) 008 [arXiv:1503.04809] [InSPIRE].

[79] S. Gukov, D. Pei, P. Putrov and C. Vafa, BPS spectra and 3-manifold invariants, J. Knot Theor. Ramifications 29 (2020) 2040003 [arXiv:1701.06567] [inSPIRE].

[80] L.F. Alday, P. Benetti Genolini, M. Bullimore and M. van Loon, Refined $3 d-3 d$ Correspondence, JHEP 04 (2017) 170 [arXiv:1702.05045] [INSPIRE].

[81] J. Eckhard, H. Kim, S. Schäfer-Nameki and B. Willett, Higher-Form Symmetries, Bethe Vacua, and the 3d-3d Correspondence, JHEP 01 (2020) 101 [arXiv:1910.14086] [INSPIRE].

[82] F. Benini and P. Milan, Black Holes in $4 D \mathcal{N}=4$ Super-Yang-Mills Field Theory, Phys. Rev. X 10 (2020) 021037 [arXiv: 1812.09613] [INSPIRE].

[83] S. Choi, C. Hwang and S. Kim, Quantum vortices, M2-branes and black holes, arXiv: 1908.02470 [INSPIRE].

[84] A. Cabo-Bizet and S. Murthy, Supersymmetric phases of $4 d \mathcal{N}=4$ SYM at large N, JHEP 09 (2020) 184 [arXiv: 1909.09597] [INSPIRE].

[85] S. Choi and C. Hwang, Universal 3d Cardy Block and Black Hole Entropy, JHEP 03 (2020) 068 [arXiv: 1911.01448] [INSPIRE].

[86] A. Arabi Ardehali, J. Hong and J.T. Liu, Asymptotic growth of the $4 d \mathcal{N}=4$ index and partially deconfined phases, JHEP 07 (2020) 073 [arXiv: 1912.04169] [INSPIRE].

[87] S. Murthy, The growth of the $\frac{1}{16}-B P S$ index in $4 d \mathcal{N}=4 S Y M$, arXiv:2005.10843 [INSPIRE].

[88] P. Agarwal, S. Choi, J. Kim, S. Kim and J. Nahmgoong, AdS black holes and finite $N$ indices, Phys. Rev. D 103 (2021) 126006 [arXiv:2005.11240] [InSPIRE].

[89] C. Copetti, A. Grassi, Z. Komargodski and L. Tizzano, Delayed Deconfinement and the Hawking-Page Transition, arXiv:2008.04950 [INSPIRE].

[90] A. Cabo-Bizet, From multi-gravitons to Black holes: The role of complex saddles, arXiv: 2012.04815 [INSPIRE].

[91] J. Yagi, 3d TQFT from 6d SCFT, JHEP 08 (2013) 017 [arXiv: 1305.0291] [INSPIRE].

[92] S. Lee and M. Yamazaki, 3d Chern-Simons Theory from M5-branes, JHEP 12 (2013) 035 [arXiv: 1305.2429] [INSPIRE].

[93] C. Cordova and D.L. Jafferis, Complex Chern-Simons from M5-branes on the Squashed Three-Sphere, JHEP 11 (2017) 119 [arXiv:1305.2891] [INSPIRE]. 
[94] T. Dimofte, Complex Chern-Simons Theory at Level $k$ via the $3 d-3 d$ Correspondence, Commun. Math. Phys. 339 (2015) 619 [arXiv:1409.0857] [InSPIRE].

[95] W.D. Neumann and D. Zagier, Volumes of hyperbolic three-manifolds, Topology 24 (1985) 307.

[96] T. Yoshida, The $\eta$-invariant of hyperbolic 3-manifolds, Invent. Math. 81 (1985) 473.

[97] F. Benini and A. Zaffaroni, Supersymmetric partition functions on Riemann surfaces, Proc. Symp. Pure Math. 96 (2017) 13 [arXiv:1605.06120] [InSPIRE].

[98] R. Meyerhoff, Density of the Chern-Simons invariant for hyperbolic 3-manifolds, in London Mathematical Society Lecture Note Series. Vol. 112: Low-dimensional topology and Kleinian groups, Cambridge University Press, Cambridge U.K. (1988), pg. 217.

[99] E. Witten, Quantization of Chern-Simons Gauge Theory With Complex Gauge Group, Commun. Math. Phys. 137 (1991) 29 [inSPIRE]. 